\title{
INFINITELY DIVISIBLE DISTRIBUTIONS IN INTEGER-VALUED GARCH MODELS
}

\author{
E. Gonçalves ${ }^{1}$, N. Mendes-Lopes, F. Silva \\ CMUC, Department of Mathematics, University of Coimbra
}

\begin{abstract}
The aim of this paper is to propose an integer-valued stochastic process with conditional marginal distribution belonging to the general class of infinitely divisible discrete probability laws.

With this proposal, we introduce a wide class of models for count time series that includes, in particular, the Poisson INGARCH model (Ferland et al., 2006) and the negative binomial and generalized Poisson INGARCH models, introduced by Zhu in 2011 and 2012a, respectively.

The main probabilistic analysis of this process is here developed. Precisely, first and second order stationarity conditions are derived as well as the autocorrelation function. The existence of a strictly stationary and ergodic solution is established in a subclass including the Poisson and generalized Poisson INGARCH models.
\end{abstract}

Keywords: integer-valued time series, GARCH model, infinitely divisible discrete probability laws, compound Poisson distributions.

\section{Introduction}

For several years, the studies of time series models were dominated by real-valued stochastic processes. However, many authors have underlined that such models do not give an adequate answer for integer-valued time series. For instance, when we deal with low dimension samples disregarding the nature of the data it leads, in general, to senseless results as the asymptotic behavior of the corresponding statistical parameters or distributions is not available. Since this type of time series is quite common in various contexts and scientific fields, including medicine, economics, finance, epidemiology, tourism and queuing systems, over the past few years different approaches to analyze and estimate this kind of data have been presented in literature.

Taking as reference the study associated with ARMA models, the general family of integervalued ARMA models (or briefly, INARMA) has been introduced and developed with the scalar multiplication replaced by an integer-valued operator with analogous properties, called thinning operation (Weiß, 2008, for a survey). Several integer-valued models have been introduced like bilinear models (Doukhan et al., 2006, Drost et al., 2008) or conditionally heteroscedastic ones (Ferland et al., 2006, Zhu et al., 2010, Zhu, 2011, 2012a, among others).

The introduction of the conditionally heteroscedastic models seems to be very useful as to deal with series of counts under hypotheses of homogeneous variance may be unrealistic in many important situations, like it is observed in Ferland et al. (2006). They present, in particular, a real sample in which the change of the series variability is evident, namely the time series of the number of cases of campylobacteriosis infections from January 1990 to the end of October 2000 in the north of the Province of Québec. To take into account these features, they propose an integer-valued process, analogous to the GARCH model introduced by Bollerslev in 1986 but with Poisson deviates, denoted $\operatorname{INGARCH}(p, q)$ model and defined as

$$
\left\{\begin{array}{l}
X_{t} \mid \underline{X}_{t-1}: \mathcal{P}\left(\lambda_{t}\right), \quad \forall t \in \mathbb{Z} \\
\lambda_{t}=\alpha_{0}+\sum_{i=1}^{p} \alpha_{i} X_{t-i}+\sum_{j=1}^{q} \beta_{j} \lambda_{t-j}
\end{array}\right.
$$

with $\alpha_{0}>0, \alpha_{i} \geq 0, \beta_{j} \geq 0, i=1, \ldots, p, j=1, \ldots, q, \underline{X}_{t-1}$ the $\sigma$-field generated by $\left\{X_{t-i}, i \geq 1\right\}$ and where $\mathcal{P}(\lambda)$ is the Poisson distribution with parameter $\lambda$. This model was further investigated by other authors, such as Weiß (2009) who derived a set of equations from which the variance

\footnotetext{
${ }^{1}$ Corresponding author: Esmeralda Gonçalves E-mail: esmerald@mat.uc.pt CMUC, Department of Mathematics, University of Coimbra, Apartado 3008, EC Santa Cruz, 3001-501 Coimbra, Portugal.
} 
and the autocorrelation function can be obtained, or Fokianos, Rahbek and Tjøtheim (2009) that considered geometric ergodicity and likelihood-based inference.

Replacing the distribution of deviates by other particular discrete ones, like negative binomial or generalized Poisson, analogous integer-valued GARCH models have been proposed and studied (Zhu, 2011, 2012a). Considering the class of mixed Poisson distributions, Fokianos and Christou (2012) generalize Poisson framework and obtain, under some hypotheses, a negative binomial integer-valued GARCH model.

With the aim of enlarging and unifying this class of INGARCH models, we introduce in this paper an integer-valued process with general infinitely divisible deviates. Thus, taking into account the equivalence between discrete infinitely divisible and compound Poisson distributions (Steutel and van Harn, 2004) we define this conditional distribution using the general formulation of the characteristic function of a compound Poisson law. With this new definition a wide set of probability distributions for deviates is considered which includes, in particular, those related to the models referred above. Precisely, we may identify this set with the family of the probability distributions of a Poissonian random sum of independent variables with discrete distribution. For this general class of integer-valued processes, different kinds of stationarity are analyzed as well as the property of ergodicity.

The remainder of the paper is organized as follows. In Section 2 we define the model and present important particular cases as the geometric Poisson integer-valued GARCH model. In Section 3 we establish first and second order stationarity conditions of the model and deduce the corresponding autocorrelation function. A necessary and sufficient condition for the existence of a strictly stationary and ergodic solution for a subclass of these models is also obtained. Some concluding remarks and future developments are given in Section 4. In Appendix A we collect some notation and assumptions and in Appendices B and C we detail some matrices and auxiliary calculations.

\section{Definition of the model}

Let $X=\left(X_{t}, t \in \mathbb{Z}\right)$ be a stochastic process with values in $\mathbb{N}_{0}$ and, for any $t \in \mathbb{Z}$, let $\underline{X}_{t-1}$ be the $\sigma$-field generated by $\left\{X_{t-j}, j \geq 1\right\}$.

Definition 2.1 (CP-INGARCH(p,q) model) The process $X$ is said to satisfy a Compound Poisson INteger-valued GARCH model with orders $p$ and $q(p, q \in \mathbb{N})$ if, $\forall t \in \mathbb{Z}$, the characteristic function of $X_{t} \mid \underline{X}_{t-1}$ is given by

$$
\left\{\begin{array}{l}
\Phi_{X_{t} \mid \underline{X}_{t-1}}(u)=e^{i \frac{\lambda_{t}}{\varphi_{t}^{\prime}(0)}\left[\varphi_{t}(u)-1\right]}, \quad u \in \mathbb{R} \\
E\left(X_{t} \mid \underline{X}_{t-1}\right)=\lambda_{t}=\alpha_{0}+\sum_{j=1}^{p} \alpha_{j} X_{t-j}+\sum_{k=1}^{q} \beta_{k} \lambda_{t-k}
\end{array}\right.
$$

for some constants $\alpha_{0}>0, \alpha_{j} \geq 0(j=1, \ldots, p), \beta_{k} \geq 0(k=1, \ldots, q)$, and where $\left(\varphi_{t}, t \in \mathbb{Z}\right)$ is a family of characteristic functions on $\mathbb{R}, \underline{X}_{t-1}$-measurable associated to a family of discrete laws with support $\mathbb{N}_{0}$ and finite mean. $i$ denotes the imaginary unit.

As $\varphi_{t}, t \in \mathbb{Z}$, is the characteristic function of a discrete law with support $\mathbb{N}_{0}$ and finite mean, the derivative of $\varphi_{t}$ at $u=0, \varphi_{t}^{\prime}(0)$, exists and is nonzero.

The designation of compound Poisson integer-valued GARCH model follows from the formulation of the characteristic function of the conditional distribution of $X_{t}$ as the characteristic function of a compound Poisson distribution is expressed as $\Phi(u)=e^{\lambda[\varphi(u)-1]}, u \in \mathbb{R}$, where $\varphi$ is a characteristic function and $\lambda$ a strictly positive real number (Steutel and van Harn, 2004).

In the previous definition, if $\beta_{k}=0, k=1, \ldots, q$, the $\operatorname{CP-} \operatorname{INGARCH}(p, q)$ model is simply denoted CP-INARCH $(p)$.

Observation 2.1 1 . As the conditional distribution of $X_{t}$ is a discrete compound Poisson law with support $\mathbb{N}_{0}$ then, $\forall t \in \mathbb{Z}$ and conditionally to $\underline{X}_{t-1}, X_{t}$ can be identified in distribution 


$$
X_{t} \stackrel{d}{=} \sum_{j=1}^{N_{t}} X_{t, j},
$$

where $N_{t}$ follows a Poisson law with parameter $\lambda_{t}^{*}=i \lambda_{t} / \varphi_{t}^{\prime}(0)$, and $X_{t, 1}, \ldots, X_{t, N_{t}}$ are discrete independent random variables, with support contained in $\mathbb{N}_{0}$, independent of $N_{t}$ and having characteristic function $\varphi_{t}$ with first derivative at zero, that is, with finite mean. We note that the characteristic functions $\varphi_{t}$ (respectively, the associated laws of probability) being $\underline{X}_{t-1}$-measurable may be random functions (respectively, random measures). This means that $\varphi_{t}$ may depend on the previous observations of the process, as we shall see in the following examples.

2. Consider $\left(\varphi_{t}, t \in \mathbb{Z}\right)$ derivable at zero up to order 2. From the definition of the model and using the relationship between the characteristic function and the moments of a distribution we have

$$
V\left(X_{t} \mid \underline{X}_{t-1}\right)=-\Phi_{X_{t} \mid \underline{X}_{t-1}^{\prime \prime}}(0)-\lambda_{t}^{2}=-i \frac{\varphi_{t}^{\prime \prime}(0)}{\varphi_{t}^{\prime}(0)} \lambda_{t} .
$$

3. Let us consider the polynomials

$$
A(L)=\alpha_{1} L+\ldots+\alpha_{p} L^{p} \quad \text { and } \quad B(L)=1-\beta_{1} L-\ldots-\beta_{q} L^{q},
$$

where $L$ is the backshift operator. To ensure the existence of the inverse of $B(L)$ we suppose that the roots of $B(z)=0$ lie outside the unit circle which, for non-negative $\beta_{j}$, is equivalent to the hypothesis $\mathbf{H 1}: \sum_{j=1}^{q} \beta_{j}<1$. Thus, under this assumption, we can rewrite the conditional expectation of the model (1) in the form

$$
B(L) \lambda_{t}=\alpha_{0}+A(L) X_{t} \Leftrightarrow \lambda_{t}=\alpha_{0} B^{-1}(1)+H(L) X_{t}
$$

with $H(L)=B^{-1}(L) A(L)=\sum_{j=1}^{\infty} \psi_{j} L^{j}$, where $\psi_{j}$ is the coefficient of $z^{j}$ in the Taylor expansion of the rational function $A(z) / B(z)$ in the neighbourhood of 0 , i.e.,

$$
\lambda_{t}=\alpha_{0} B^{-1}(1)+\sum_{j=1}^{\infty} \psi_{j} X_{t-j}
$$

which expresses a CP-INARCH( $\infty)$ representation of the model in study.

Observation 2.2 The model (1) includes several models already studied in the literature. Indeed,

1. Ferland et al. (2006) introduced the model INGARCH(p,q) mentioned in Section 1, which corresponds to the present model considering $\varphi_{t}$ the characteristic function of the Dirac's law concentrated in $\{1\}, \delta(1)$.

2. In Zhu (2011) the NB-INGARCH( $p, q)$ model was studied, in analogy with Ferland's model but where the $X_{t} \mid \underline{X}_{t-1}$ distribution is the negative binomial law with parameters $\left(r, p_{t}\right)$ with $p_{t}=\frac{1}{1+\lambda_{t}}$ and $r \in \mathbb{N}$. Considering, in the model (1), $\varphi_{t}$ the characteristic function of a logarithmic distribution with parameter $1-\exp \left(-\lambda_{t}^{*} / r\right)$ we recover, unless a scale factor, the previous model.

3. Zhu (2012a) proposed the GP-INGARCH( $p, q)$ model taking as distribution of $X_{t} \mid \underline{X}_{t-1}$ the generalized Poisson law with parameters $\left(\lambda_{t}^{*}, \kappa\right)$ where $\lambda_{t}^{*}=(1-\kappa) \lambda_{t}$ and $0<\kappa<1$. This model results from the model (1) considering $\varphi_{t}$ the characteristic function of the Borel's law with parameter $\kappa$ (Consul and Famoye, 2006, Weiß, 2008).

4. Xu et al. (2012) presented the NB-DINARCH(p) model that differs from the NB-INARCH(p) model of Zhu because the parameters are $\left(r_{t}, p^{*}\right)$ with $r_{t}=\frac{p^{*}}{1-p^{*}} \lambda_{t}$ and $\left.p^{*} \in\right] 0,1[$. So, this model results from model (1) considering $\varphi_{t}$ the characteristic function of a logarithmic distribution with parameter $p^{*}$ and $\lambda_{t}^{*}=-r_{t} \ln \left(1-p^{*}\right)$. 
The examples presented in Observation 2.2 show that $\varphi_{t}$ may be a random characteristic function (case 2) or a deterministic one (cases 1, 3 and 4). In fact, the parameter involved in the characteristic function $\varphi_{t}$ in the NB-INGARCH model depends on the previous observations of the model, via $\lambda_{t}$, while in the other models that parameter is constant when $t$ varies.

Moreover a wide class of processes is included in model (1). The following examples show how to obtain this kind of processes and also a particular situation where $\left(\varphi_{t}\right)$ is a family of dependent on $t$ deterministic characteristic functions (example 2.1.4).

Example 2.1 Let us take into account the representation stated in (2).

1. Let us consider independent random variables $\left(X_{t, j}, t \in \mathbb{Z}\right)$ following a geometric law with parameter $p_{t}=\frac{r}{r+\lambda_{t}}$ and $r>0$ arbitrarily fixed, that is, $\varphi_{t}(u)=\frac{p_{t} e^{i u}}{1-\left(1-p_{t}\right) e^{i u}}, u \in \mathbb{R}, t \in \mathbb{Z}$. If $N_{t}$ is a random variable independent of $X_{t, j}$ and following a Poisson law with parameter $r$ then, the process $X_{t}=\sum_{j=1}^{N_{t}} X_{t, j}$ satisfies, unless an additive parameter $r$, the model (1) where the $X_{t} \mid \underline{X}_{t-1}$ law is the geometric Poisson (also called Pólya-Aeppli) distribution with parameters $\left(r, p_{t}\right)$ (Özel and Inal, 2010). In this case, the model will be denoted by GEOMP-INGARCH $(p, q)$ model.

2. Considering the characteristic functions $\varphi_{t}(u)=\sum_{n=2}^{\infty} \frac{\Lambda(n) n^{-\left(i u+s_{t}\right)}}{\ln \zeta\left(s_{t}\right) \ln n}, u \in \mathbb{R}, t \in \mathbb{Z}$, where $\Lambda$ and $\zeta$ are the Mangoldt and the Riemann zeta functions, respectively, and $\lambda_{t}^{*}=\ln \zeta\left(s_{t}\right)$, we obtain the model (1) where the $X_{t} \mid \underline{X}_{t-1}$ distribution is the discrete Pareto law with parameter $s_{t}$ (Steutel and van Harn, 2004, Gut, 2006).

3. Let us consider independent and identically distributed random variables $\left(X_{t, j}, t \in \mathbb{Z}\right)$ following any discrete law with finite mean, constant parameters and support contained in $\mathbb{N}_{0}$. Taking $N_{t}$ independent of $X_{t, j}$ and following a Poisson law with parameter $\frac{\lambda_{t}}{E\left(X_{t, j}\right)}$, the resulting process $X$ follows the model (1). For instance, we may consider the geometric distribution with parameter $\left.p^{*} \in\right] 0,1\left[\right.$ for $X_{t, j}$ and the parameter $p^{*} \lambda_{t}$ in the Poisson law of $N_{t}$. In this case, the model will be denoted by GEOMP2-INGARCH(p,q) model.

4. If $\left(X_{t, j}, t \in \mathbb{Z}\right)$ are independent random variables following the binomial distribution with parameters $r \in \mathbb{N}$ and $e^{-|t|}$, that is, $\varphi_{t}(u)=\left(e^{i u-|t|}+1-e^{-|t|}\right)^{r}, u \in \mathbb{R}, t \in \mathbb{Z}$, and $N_{t}$ is an independent of $X_{t, j}$ random variable following $\mathcal{P}\left(\frac{\lambda_{t}}{r e^{-|t|}}\right)$ then $X$ satisfies model (1).

Figure 1 illustrates the trajectories and the basic descriptives of INGARCH $(1,1)$ models, with $\alpha_{0}=10, \alpha_{1}=0.4, \beta_{1}=0.5$, and considering Poisson, negative binomial and geometric Poisson deviates, the last one introduced in Example 2.1.1 using the methodology here proposed. We point out the differences observed in the trajectories and also the strong volatility and large kurtosis values recorded, namely in the GEOMP-INGARCH case.

\section{$3 \quad$ Stationarity properties}

In time series modeling, to evaluate stability properties over time is important in statistical developments, in particular to reach good forecasts. The study of the stationarity of such models is thus a basic issue in their probabilistic analysis and is the subject of this section.

\subsection{First order stationarity}

The following theorem gives a necessary and sufficient condition for the first order stationarity of the general model introduced in (1). It includes in particular the analogous property of Ferland et al. (2006) and Zhu (2011, 2012a). The proof of this result is done with the same arguments used in Proposition 1 of Ferland et al. (2006), so we omit the details. 

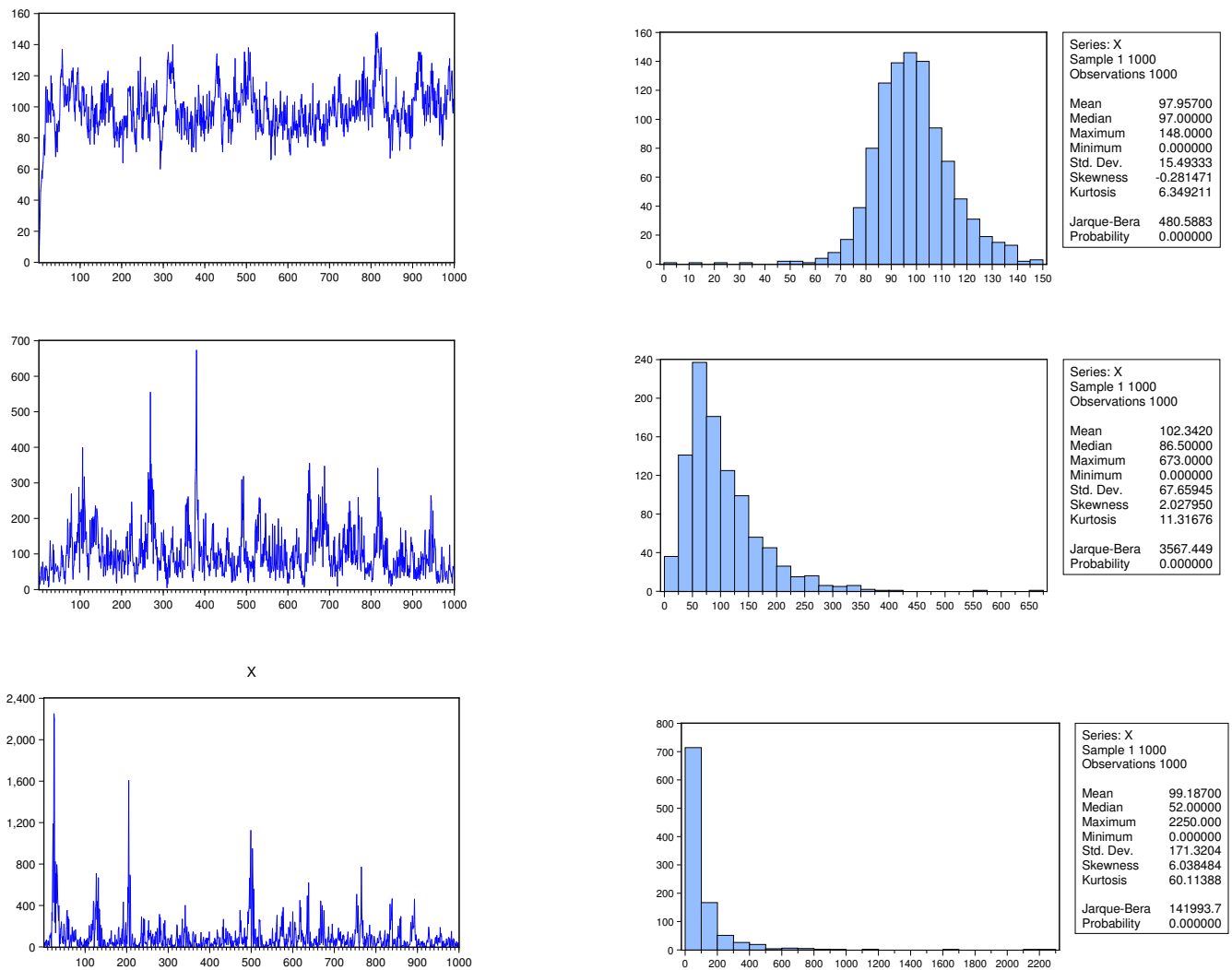

Figure 1: Trajectories and descriptives of $\operatorname{INGARCH}(1,1)$ models with Poisson (on top), negative binomial (middle) and geometric Poisson (below) conditional laws $\left(\alpha_{0}=10, \alpha_{1}=0.4\right.$, $\left.\beta_{1}=0.5\right)$.

Theorem 3.1 Let $\left(X_{t}, t \in \mathbb{Z}\right)$ be a process satisfying the CP-INGARCH $(p, q)$ model. This process is first order stationary if and only if $\sum_{i=1}^{p} \alpha_{i}+\sum_{j=1}^{q} \beta_{j}<1$.

Observation 3.1 As a consequence of the previous theorem, if $\sum_{i=1}^{p} \alpha_{i}+\sum_{j=1}^{q} \beta_{j}<1$, the processes $\left(X_{t}\right)$ and $\left(\lambda_{t}\right)$ are both first order stationary and we have

$$
E\left(X_{t}\right)=E\left(\lambda_{t}\right)=\mu=\frac{\alpha_{0}}{1-\sum_{i=1}^{p} \alpha_{i}-\sum_{j=1}^{q} \beta_{j}} .
$$

\subsection{Second order stationarity}

To develop the study on the second order stationarity of model (1) we assume that the family of characteristic functions $\left(\varphi_{t}, t \in \mathbb{Z}\right)$ is derivable at zero up to order 2 in order to assure the existence of the corresponding distribution variance. The general class of models considered and the complexity in the study of the second order stationarity in this class leads us to fix ourselves in the subclass of CP-INGARCH $(p, q)$ models for which $\varphi_{t}$ satisfies the hypothesis

$$
\text { H2 : } \frac{V\left(X_{t} \mid \underline{X}_{t-1}\right)}{E\left(X_{t} \mid \underline{X}_{t-1}\right)}=-i \frac{\varphi_{t}^{\prime \prime}(0)}{\varphi_{t}^{\prime}(0)}=v_{0}+v_{1} \lambda_{t},
$$

with $v_{0} \geq 0, v_{1} \geq 0$, not simultaneously zero. Despite the restriction, it can be shown that a quite general subclass is considered, containing both random and deterministic characteristic functions since they have the general form $\varphi_{t}(u)=\exp \zeta(t) \int k(u)^{i\left(v_{0}+v_{1} \lambda_{t}\right)} d u+\delta(t)$, with $k^{\prime}(0) / k(0)=1$, $u \in \mathbb{R}$. Let us note that all the examples presented in the observation 2.2 belong to this subclass 
of models. Indeed, the INGARCH with Poisson deviates, the GP-INGARCH and the NB-DINARCH models satisfy the hypothesis $\boldsymbol{H} 2$ with $v_{1}=0$ and $v_{0}=1,(1-\kappa)^{-2},\left(1-p^{*}\right)^{-1}$, respectively, and the NB-INGARCH model with $v_{0}=1$ and $v_{1}=\frac{1}{r}$. We also point out that, using the methodology proposed in this paper, it is easy to build other models that satisfy $\boldsymbol{H}$ 2. For instance, the proposed GEOMP-INGARCH model satisfies $\boldsymbol{H} 2$ with $v_{0}=1$ and $v_{1}=\frac{2}{r}$.

We begin by establishing a result which reveals to be useful to obtain a sufficient condition of second order stationarity. We note that the approach here followed is different from those presented in literature for the integer-valued models. It begins by stating a vectorial state space representation of $X$ from which a sufficient condition of weak stationarity is naturally deduced.

Proposition 3.1 Let $X$ be a first order stationary process following a CP-INGARCH(p,q) model such that $\mathbf{H 2}$ is satisfied. The vector $W_{t}, t \in \mathbb{Z}$, of dimension $p+q-1$ given by

$$
W_{t}=\left[\begin{array}{c}
E\left(X_{t}^{2}\right) \\
E\left(X_{t} X_{t-1}\right) \\
\cdots \\
E\left(X_{t} X_{t-(p-1)}\right) \\
E\left(\lambda_{t} \lambda_{t-1}\right) \\
\cdots \\
E\left(\lambda_{t} \lambda_{t-(q-1)}\right)
\end{array}\right]
$$

satisfies an autoregressive equation of order $\max (p, q)$ :

$$
W_{t}=B_{0}+\sum_{k=1}^{\max (p, q)} B_{k} W_{t-k},
$$

where $B_{0}$ is a real vector of dimension $p+q-1$ and $B_{k}(k=1, \ldots, \max (p, q))$ are real squared matrices of order $p+q-1$.

Proof. Let us start by noting that $E\left(X_{t}^{2}\right), E\left(X_{t} X_{t-k}\right)$ and $E\left(\lambda_{t} \lambda_{t-k}\right)$ are not necessarily finite but, as we have positive and measurable functions, the involved integrals exist.

For simplicity, we focus on the case $p=q$. The other cases can be obtained from this one setting additional parameters to 0 . We begin to calculate $E\left(X_{t}^{2}\right)$ for any $t \in \mathbb{Z}$. We have $E\left(X_{t}^{2}\right)=E\left[E\left(X_{t}^{2} \mid \underline{X}_{t-1}\right)\right]$ and

$$
\begin{aligned}
& E\left(X_{t}^{2} \mid \underline{X}_{t-1}\right)=V\left(X_{t} \mid \underline{X}_{t-1}\right)+\left[E\left(X_{t} \mid \underline{X}_{t-1}\right)\right]^{2}=v_{0} \lambda_{t}+\left(1+v_{1}\right) \lambda_{t}^{2} \\
& =v_{0} \alpha_{0}+\left(1+v_{1}\right) \alpha_{0}^{2}+\left[v_{0}+2 \alpha_{0}\left(1+v_{1}\right)\right]\left(\sum_{i=1}^{p} \alpha_{i} X_{t-i}+\sum_{j=1}^{p} \beta_{j} \lambda_{t-j}\right)+\left(1+v_{1}\right)\left[\sum_{i=1}^{p} \alpha_{i}^{2} X_{t-i}^{2}\right. \\
& \left.\quad+\sum_{\substack{i, j=1 \\
i \neq j}}^{p} \alpha_{i} \alpha_{j} X_{t-i} X_{t-j}+2 \sum_{i=1}^{p} \sum_{j=1}^{p} \alpha_{i} \beta_{j} \lambda_{t-j} X_{t-i}+\sum_{i=1}^{p} \beta_{i}^{2} \lambda_{t-i}^{2}+\sum_{\substack{i, j=1 \\
i \neq j}}^{p} \beta_{i} \beta_{j} \lambda_{t-i} \lambda_{t-j}\right] .
\end{aligned}
$$

So, using the first order stationary hypothesis, we conclude

$$
\begin{aligned}
E\left(X_{t}^{2}\right)=\widetilde{C}+\left(1+v_{1}\right)\left[\sum_{i=1}^{p} \alpha_{i}^{2} E\left(X_{t-i}^{2}\right)+\sum_{\substack{i, j=1 \\
i \neq j}}^{p} \alpha_{i} \alpha_{j} E\left(X_{t-i} X_{t-j}\right)\right. \\
\left.+2 \sum_{i, j=1}^{p} \alpha_{i} \beta_{j} E\left(X_{t-i} \lambda_{t-j}\right)+\sum_{\substack{i=1 \\
i}}^{p} \beta_{i}^{2} E\left(\lambda_{t-i}^{2}\right)+\sum_{\substack{i, j=1 \\
i \neq j}}^{p} \beta_{i} \beta_{j} E\left(\lambda_{t-i} \lambda_{t-j}\right)\right] \\
=C+\left(1+v_{1}\right)\left[\sum_{i=1}^{p}\left(\alpha_{i}^{2}+\frac{2 \alpha_{i} \beta_{i}+\beta_{i}^{2}}{1+v_{1}}\right) E\left(X_{t-i}^{2}\right)\right.
\end{aligned}
$$




$$
\left.+2 \sum_{i=1}^{p-1} \sum_{j=i+1}^{p} \alpha_{j}\left(\alpha_{i}+\beta_{i}\right) E\left(X_{t-i} X_{t-j}\right)+2 \sum_{i=1}^{p-1} \sum_{j=i+1}^{p} \beta_{j}\left(\alpha_{i}+\beta_{i}\right) E\left(\lambda_{t-i} \lambda_{t-j}\right)\right]
$$

where $\widetilde{C}=v_{0} \mu+\left(1+v_{1}\right)\left[2 \alpha_{0} \mu-\alpha_{0}^{2}\right]$ and $C=\widetilde{C}-v_{0} \mu \sum_{i=1}^{p}\left(2 \alpha_{i} \beta_{i}+\beta_{i}^{2}\right)$ are positive constants and independent of $t$, and where we took into account the following facts:

$$
E\left(X_{t-i} \lambda_{t-j}\right)=\left\{\begin{array}{ll}
E\left(\lambda_{t-i} \lambda_{t-j}\right), & \text { if } j \geq i \\
E\left(X_{t-i} X_{t-j}\right), & \text { if } j<i
\end{array}, \quad E\left(\lambda_{t}^{2}\right)=\frac{E\left(X_{t}^{2}\right)-v_{0} \mu}{1+v_{1}}, \quad \mu-\alpha_{0}=\mu \sum_{i=1}^{p}\left(\alpha_{i}+\beta_{i}\right) .\right.
$$

We observe from this second equality that $X$ is a $L^{2}$ process if and only if the same happens to $\lambda$.

On the other hand, when $k \geq 1$,

$$
\begin{array}{r}
E\left(X_{t} X_{t-k}\right)=E\left[E\left(X_{t} \mid \underline{X}_{t-1}\right) X_{t-k}\right]=E\left(\left[\alpha_{0}+\sum_{i=1}^{p} \alpha_{i} X_{t-i}+\sum_{j=1}^{p} \beta_{j} \lambda_{t-j}\right] X_{t-k}\right) \\
=\left[\alpha_{0}-\frac{v_{0} \beta_{k}}{1+v_{1}}\right] \mu+\left[\alpha_{k}+\frac{\beta_{k}}{1+v_{1}}\right] E\left(X_{t-k}^{2}\right)+\sum_{i=k+1}^{p} \beta_{i} E\left(\lambda_{t-i} \lambda_{t-k}\right) \\
+\sum_{i=1}^{k-1}\left(\alpha_{i}+\beta_{i}\right) E\left(X_{t-i} X_{t-k}\right)+\sum_{i=k+1}^{p} \alpha_{i} E\left(X_{t-i} X_{t-k}\right) .
\end{array}
$$

Similarly we obtain, for $k \geq 1$,

$$
\begin{array}{r}
E\left(\lambda_{t} \lambda_{t-k}\right)=\left[\alpha_{0}-\frac{v_{0}\left(\alpha_{k}+\beta_{k}\right)}{1+v_{1}}\right] \mu+\frac{\alpha_{k}+\beta_{k}}{1+v_{1}} E\left(X_{t-k}^{2}\right)+\sum_{i=k+1}^{p} \alpha_{i} E\left(X_{t-i} X_{t-k}\right) \\
+\sum_{i=1}^{k-1}\left(\alpha_{i}+\beta_{i}\right) E\left(\lambda_{t-i} \lambda_{t-k}\right)+\sum_{i=k+1}^{p} \beta_{i} E\left(\lambda_{t-i} \lambda_{t-k}\right) .
\end{array}
$$

Using the above expressions it is clear that $W_{t}=B_{0}+\sum_{k=1}^{p} B_{k} W_{t-k}$, with $B_{0}$ the vector and $B_{k}(k=1, \ldots, p)$ the matrices presented in Appendix B.

For a CP-INARCH $(p)$ model, in which we consider, for simplicity, $q=1$ and $\beta_{1}=0$, the previous result assumes the form presented in the following corollary.

Corollary 3.1 Let $X$ be a first order stationary process following a CP-INARCH(p) model such that the hypothesis $\boldsymbol{H} 2$ is satisfied. The vector $W_{t}=\left(E\left(X_{t}^{2}\right), E\left(X_{t} X_{t-1}\right), \ldots, E\left(X_{t} X_{t-(p-1)}\right)\right)$, $t \in \mathbb{Z}$, follows an autoregressive equation of order $p: W_{t}=B_{0}+\sum_{k=1}^{p} B_{k} W_{t-k}$, where the vector $B_{0}=\left(v_{0} \mu+\alpha_{0}\left(1+v_{1}\right)\left(2 \mu-\alpha_{0}\right), \alpha_{0} \mu, \ldots, \alpha_{0} \mu\right)$ is of dimension $p$ and $B_{k}(k=1, \ldots, p)$ are squared matrices of order $p$ with generic element $b_{i j}^{(k)}$ given by:

- $\operatorname{row} i=1$ :

$$
b_{1 j}^{(k)}= \begin{cases}\left(1+v_{1}\right) \alpha_{k}^{2}, & \text { if } j=1 \\ 2\left(1+v_{1}\right) \alpha_{k} \alpha_{j+k-1}, & \text { if } j=2, \ldots, p\end{cases}
$$

- $\operatorname{row} i \neq 1$ :

$$
b_{i j}^{(k)}= \begin{cases}\alpha_{j+k-1}, & \text { if } i=k+1, j=1, \ldots, p \\ \alpha_{k}, & \text { if } i=k+j, j=2, \ldots, p \\ 0, & \text { otherwise }\end{cases}
$$

where $\alpha_{i}=0$ for $i>p$

Now we can obtain a sufficient condition for weak stationarity of the process under study. 
Theorem 3.2 Let $X$ be a first order stationary process following a CP-INGARCH $(p, q)$ model such that $\mathbf{H} 2$ is satisfied. This process is weakly stationary if

$$
P(L)=I_{p+q-1}-\sum_{k=1}^{\max (p, q)} B_{k} L^{k}
$$

is a polynomial matrix such that $\operatorname{det} P(z)$ has all its roots outside the unit circle, where $I_{p+q-1}$ is the identity matrix of order $p+q-1$ and $B_{k}(k=1, \ldots, \max (p, q))$ are the squared matrices of the autoregressive equation (3). Moreover,

$$
\begin{aligned}
& \operatorname{Cov}\left(X_{t}, X_{t-j}\right)=e_{j+1}[P(1)]^{-1} B_{0}-\mu^{2}, \quad j=0, \ldots, p-1, \\
& \operatorname{Cov}\left(\lambda_{t}, \lambda_{t-j}\right)=e_{p+j}[P(1)]^{-1} B_{0}-\mu^{2}, \quad j=1, \ldots, q-1,
\end{aligned}
$$

with $e_{j}$ the order $j$ row of the identity matrix.

Proof. Without loss of generality, let us consider $p \geq q$. As $\operatorname{det} P(z)$ has all roots outside the unit circle then $\operatorname{det} P(1)=\operatorname{det}\left(I_{p+q-1}-\sum_{k=1}^{p} B_{k}\right) \neq 0$, that is, $P(1)$ is an invertible matrix. Thus, we obtain

$$
\begin{aligned}
& W_{t}=B_{0}+\sum_{k=1}^{p} B_{k} W_{t-k} \Leftrightarrow\left(I_{p+q-1}-\sum_{k=1}^{p} B_{k} L^{k}\right) W_{t}=B_{0} \\
\Leftrightarrow & W_{t}-[P(1)]^{-1} B_{0}=\sum_{k=1}^{p} B_{k}\left(W_{t-k}-[P(1)]^{-1} B_{0}\right) .
\end{aligned}
$$

So the last equation shows that the sequence $\left(W_{t}-[P(1)]^{-1} B_{0}\right)$ satisfies an homogeneous linear recurrence equation. From Goldberg (1958) we conclude

$$
\lim _{t} W_{t}=[P(1)]^{-1} B_{0},
$$

i.e., the solution of the equation is asymptotically independent of $t$. As $W_{t}$ is asymptotically independent of $t$ then, from the definition of $W_{t}$, the weak stationarity of $\left(X_{t}\right)$ and $\left(\lambda_{t}\right)$ follows.

For a first order stationary CP-INGARCH $(1,1)$ process we have the following weak stationarity characterization.

Theorem 3.3 Consider a first order stationary CP-INGARCH(1,1) model satisfying H2. A necessary and sufficient condition for weak stationarity is $\left(\alpha_{1}+\beta_{1}\right)^{2}+v_{1} \alpha_{1}^{2}<1$.

Proof. From expression (4), we obtain, in this particular case, the non-homogeneous difference equation of first order $E\left(X_{t}^{2}\right)-\left[\left(\alpha_{1}+\beta_{1}\right)^{2}+v_{1} \alpha_{1}^{2}\right] E\left(X_{t-1}^{2}\right)=C$, where $C=v_{0} \mu+\left(1+v_{1}\right)\left[2 \alpha_{0} \mu-\alpha_{0}^{2}\right]$ $-v_{0} \mu\left(\beta_{1}^{2}+2 \alpha_{1} \beta_{1}\right)>0$. If $\left(\alpha_{1}+\beta_{1}\right)^{2}+v_{1} \alpha_{1}^{2}<1$, then the above equation has an independent of $t$ solution, that is, the process is second order stationary. On the other hand, if the process is second order stationary then

$$
\left[1-\left(\alpha_{1}+\beta_{1}\right)^{2}-v_{1} \alpha_{1}^{2}\right] E\left(X_{t}^{2}\right)=C \Rightarrow\left(\alpha_{1}+\beta_{1}\right)^{2}+v_{1} \alpha_{1}^{2}<1 .
$$

Let us now develop a necessary condition of weak stationarity for a general CP-INGARCH $(p, q)$ model. We generalize the results of Zhu (2011), for a NB-INARCH $(p)$ model, extending the class of conditional distributions and considering models of general orders $(p, q)$. In that sense we consider $B=\left(b_{i j}\right)$ the squared matrix of order $p+q-2$ whose terms are, for $i=1, \ldots, p-1$, given by

$$
b_{i j}= \begin{cases}\sum_{|k-i|=j} \alpha_{k}+\beta_{i-j}, & 1 \leq j \leq i-1 \\ \alpha_{2 i}-1, & j=i \\ \sum_{|k-i|=j} \alpha_{k}, & i+1 \leq j \leq p-1 \\ \beta_{i+j}, & p \leq j \leq p+q-i-1 \\ 0, & \text { otherwise }\end{cases}
$$


and for $i=p, \ldots, p+q-2$, given by

$$
b_{i j}= \begin{cases}\alpha_{j+i-p+1}, & 1 \leq j \leq p-1 \\ \sum_{|k-i|=j} \beta_{k}+\alpha_{i-j}, & p \leq j \leq i-1 \\ \beta_{2(i-p+1)}-1, & j=i \\ \sum_{|k-i|=j} \beta_{k}, & i+1 \leq j \leq p+q-2 \\ 0, & \text { otherwise }\end{cases}
$$

where $\alpha_{i}=0$ for $i>p$ and $\beta_{j}=0$ for $j>q$. If $B^{-1}$ exists, we denote its elements by $d_{i j}$. Consider also the vector $b=\left(b_{i 0}\right)$ with components

$$
b_{i 0}=\left\{\begin{array}{ll}
\alpha_{i}+\frac{\beta_{i}}{1+v_{1}}, & i=1, \ldots, p-1 \\
\frac{\alpha_{i-p+1}+\beta_{i-p+1}}{1+v_{1}}, & i=p, \ldots, p+q-2
\end{array} .\right.
$$

Theorem 3.4 Let $X$ be a process following a CP-INGARCH( $p, q)$ model satisfying $\mathbf{H} 2$ and such that $\alpha_{0}\left(1+v_{1}\right)>v_{0}$. If the process is second order stationary then all the roots of the equation $1-C_{1} z-\ldots-C_{r} z^{r}=0$ lie outside the unit circle, with $r=\max (p, q)$ and where for $v=1, \ldots, r-1$,

$$
\begin{aligned}
C_{v} & =\left(1+v_{1}\right)\left[\alpha_{v}^{2}+\frac{2 \alpha_{v} \beta_{v}+\beta_{v}^{2}}{1+v_{1}}-2 \sum_{\substack{(i, j) \in\{1, \ldots, p\} \times\{1, \ldots, q\}: \\
j-i=v}}\left(\alpha_{i}+\beta_{i}\right) \sum_{u=1}^{p+q-2}\left(\alpha_{j} d_{v u}+\beta_{j} d_{v+r-1, u}\right) b_{u 0}\right], \\
C_{r} & =\left(1+v_{1}\right) \alpha_{r}^{2}+2 \alpha_{r} \beta_{r}+\beta_{r}^{2} .
\end{aligned}
$$

Proof. Let us start by recalling the existence of the CP-INARCH $(\infty)$ representation which results from the assumption of first order stationarity. From this representation and using the fact that $X$ is a second order stationary process we conclude the second order stationarity of $\lambda$. Let us use the notation $\gamma_{k}=E\left(X_{t} X_{t-k}\right)$ and $\widetilde{\gamma}_{k}=E\left(\lambda_{t} \lambda_{t-k}\right)$, with $k \in \mathbb{Z}$.

In what follows we use the expressions obtained for $E\left(X_{t}^{2}\right), E\left(X_{t} X_{t-k}\right)$ and $E\left(\lambda_{t} \lambda_{t-k}\right)$ in proposition 3.1 and for simplicity we restrict ourselves to the case $p=q$. From (4) we have

$$
\gamma_{0}=C+\left(1+v_{1}\right)\left[\sum_{i=1}^{p}\left(\alpha_{i}^{2}+\frac{2 \alpha_{i} \beta_{i}+\beta_{i}^{2}}{1+v_{1}}\right) \gamma_{0}+2 \sum_{v=1}^{p-1} \sum_{j-i=v}\left(\alpha_{i}+\beta_{i}\right)\left(\alpha_{j} \gamma_{v}+\beta_{j} \widetilde{\gamma}_{v}\right)\right],
$$

with $C=v_{0} \mu\left[1-\sum_{i=1}^{p}\left(2 \alpha_{i} \beta_{i}+\beta_{i}^{2}\right)\right]+\left(1+v_{1}\right)\left[2 \alpha_{0} \mu-\alpha_{0}^{2}\right]>0$ independent of $t$.

From (5) it follows that for $k=1, \ldots, p-1$,

$$
\begin{array}{r}
\gamma_{k}=\left(\alpha_{0}-\frac{v_{0} \beta_{k}}{1+v_{1}}\right) \mu+\left(\alpha_{k}+\frac{\beta_{k}}{1+v_{1}}\right) \gamma_{0}+\sum_{i=k+1}^{p} \beta_{i} \widetilde{\gamma}_{i-k}+\sum_{i=1}^{k-1}\left(\alpha_{i}+\beta_{i}\right) \gamma_{k-i}+\sum_{i=k+1}^{p} \alpha_{i} \gamma_{i-k} \\
\Leftrightarrow \gamma_{k}-\sum_{|i-k|=1} \alpha_{i} \gamma_{1}-\ldots-\sum_{|i-k|=k} \alpha_{i} \gamma_{k}-\ldots-\sum_{|i-k|=p-1} \alpha_{i} \gamma_{p-1}-\sum_{k-i=1} \beta_{i} \gamma_{1}-\ldots-\sum_{k-i=k-1} \beta_{i} \gamma_{k-1} \\
-\sum_{i-k=1} \beta_{i} \widetilde{\gamma}_{1}-\ldots-\sum_{i-k=p-k} \beta_{i} \widetilde{\gamma}_{p-k}=\left(\alpha_{0}-\frac{v_{0} \beta_{k}}{1+v_{1}}\right) \mu+\left(\alpha_{k}+\frac{\beta_{k}}{1+v_{1}}\right) \gamma_{0},
\end{array}
$$

or equivalently,

$$
\sum_{u=1}^{p-1} b_{k u} \gamma_{u}+\sum_{u=1}^{p-k} b_{k, u+p-1} \widetilde{\gamma}_{u}=-\left[\left(\alpha_{0}-\frac{v_{0} \beta_{k}}{1+v_{1}}\right) \mu+\left(\alpha_{k}+\frac{\beta_{k}}{1+v_{1}}\right) \gamma_{0}\right]
$$


with

$$
b_{k u}= \begin{cases}\sum_{|i-k|=u} \alpha_{i}+\beta_{k-u}, & 1 \leq u \leq k-1 \\ \alpha_{2 k}-1, & u=k \\ \sum_{|i-k|=u} \alpha_{i}, & k+1 \leq u \leq p-1\end{cases}
$$

and $b_{k, u+p-1}=\beta_{u+k}, u=1, \ldots, p-k$, where we consider $\alpha_{i}=\beta_{i}=0, i>p$.

Similarly we get from (6), for $k=1, \ldots, p-1$,

$$
\begin{gathered}
\widetilde{\gamma}_{k}=\left(\alpha_{0}-\frac{v_{0}\left(\alpha_{k}+\beta_{k}\right)}{1+v_{1}}\right) \mu+\frac{\alpha_{k}+\beta_{k}}{1+v_{1}} \gamma_{0}+\sum_{i=k+1}^{p} \alpha_{i} \gamma_{i-k}+\sum_{i=1}^{k-1}\left(\alpha_{i}+\beta_{i}\right) \widetilde{\gamma}_{k-i}+\sum_{i=k+1}^{p} \beta_{i} \widetilde{\gamma}_{i-k} \\
\Leftrightarrow\left(1-\beta_{2 k}\right) \widetilde{\gamma}_{k}-\sum_{|i-k|=1} \beta_{i} \widetilde{\gamma}_{1}-\ldots-\sum_{|i-k|=p-1} \beta_{i} \widetilde{\gamma}_{p-1}-\sum_{k-i=1} \alpha_{i} \widetilde{\gamma}_{1}-\ldots-\sum_{k-i=k-1} \alpha_{i} \widetilde{\gamma}_{k-1} \\
-\sum_{i-k=1} \alpha_{i} \gamma_{1}-\ldots-\sum_{i-k=p-k} \alpha_{i} \gamma_{p-k}=\left(\alpha_{0}-\frac{v_{0}\left(\alpha_{k}+\beta_{k}\right)}{1+v_{1}}\right) \mu+\frac{\alpha_{k}+\beta_{k}}{1+v_{1}} \gamma_{0},
\end{gathered}
$$

or equivalently,

$$
\sum_{u=1}^{p-k} b_{k+p-1, u} \gamma_{u}+\sum_{u=1}^{p-1} b_{k+p-1, u+p-1} \widetilde{\gamma}_{u}=-\left[\left(\alpha_{0}-\frac{v_{0}\left(\alpha_{k}+\beta_{k}\right)}{1+v_{1}}\right) \mu+\frac{\alpha_{k}+\beta_{k}}{1+v_{1}} \gamma_{0}\right]
$$

with

$$
b_{k+p-1, u+p-1}= \begin{cases}\sum_{|i-k|=u} \beta_{i}+\alpha_{k-u}, & 1 \leq u \leq k-1 \\ \beta_{2 k}-1, & u=k \\ \sum_{|i-k|=u} \beta_{i}, & k+1 \leq u \leq p-1\end{cases}
$$

and $b_{k+p-1, u}=\alpha_{u+k}, u=1, \ldots, p-k$. Let $i, j=1, \ldots, 2 p-2, B=\left(b_{i j}\right)$ and $B^{-1}=\left(d_{i j}\right)$ its inverse whose existence is a consequence of the first order stationarity (Appendix $\mathrm{C}$ ). Thus, from expressions (8) and (9) and using the invertibility of $B$ we obtain

$$
\widehat{\gamma}=\left[\begin{array}{c}
\gamma_{1} \\
\vdots \\
\gamma_{p-1} \\
\widetilde{\gamma}_{1} \\
\vdots \\
\widetilde{\gamma}_{p-1}
\end{array}\right]=-B^{-1}\left(\left[\begin{array}{c}
\alpha_{0} \mu \\
\vdots \\
\alpha_{0} \mu \\
\alpha_{0} \mu \\
\vdots \\
\alpha_{0} \mu
\end{array}\right]+v_{0} \mu\left[\begin{array}{c}
\alpha_{1}-b_{10} \\
\vdots \\
\alpha_{p-1}-b_{p-1,0} \\
-b_{p, 0} \\
\vdots \\
-b_{2 p-2,0}
\end{array}\right]+\gamma_{0} b\right)
$$

where $b$ is the vector previously introduced. So, for $l=1, \ldots, 2 p-2$,

$$
\widehat{\gamma}_{l}=-\alpha_{0} \mu \sum_{u=1}^{2 p-2} d_{l u}+v_{0} \mu\left[\sum_{u=1}^{p-1}\left(b_{u 0}-\alpha_{u}\right) d_{l u}+\sum_{u=p}^{2 p-2} b_{u 0} d_{l u}\right]-\sum_{u=1}^{2 p-2} d_{l u} b_{u 0} \gamma_{0} .
$$

Taking the last part of (7) and using the previous expression, we get

$$
\begin{aligned}
& 2 \sum_{v=1}^{p-1} \sum_{j-i=v}\left(\alpha_{i}+\beta_{i}\right)\left(\alpha_{j} \gamma_{v}+\beta_{j} \widetilde{\gamma}_{v}\right) \\
& =\widehat{C}-2 \sum_{v=1}^{p-1} \sum_{j-i=v}\left(\alpha_{i}+\beta_{i}\right)\left[\alpha_{j} \sum_{u=1}^{2 p-2} d_{v u} b_{u 0}+\beta_{j} \sum_{u=1}^{2 p-2} d_{v+p-1, u} b_{u 0}\right] \gamma_{0},
\end{aligned}
$$

where $\widehat{C}$ is a positive constant independent of $t$, as proved in Appendix C. 
Then replacing this expression in (7) we finally get

$$
\begin{array}{r}
\gamma_{0}=C_{0}+\left(1+v_{1}\right)\left[\left(\alpha_{p}^{2}+\frac{2 \alpha_{p} \beta_{p}+\beta_{p}^{2}}{1+v_{1}}\right) \gamma_{0}+\sum_{v=1}^{p-1}\left\{\left(\alpha_{v}^{2}+\frac{2 \alpha_{v} \beta_{v}+\beta_{v}^{2}}{1+v_{1}}\right)\right.\right. \\
\left.\left.-2 \sum_{j-i=v}\left(\alpha_{i}+\beta_{i}\right) \sum_{u=1}^{2 p-2}\left(\alpha_{j} d_{v u}+\beta_{j} d_{v+p-1, u}\right) b_{u 0}\right\} \gamma_{0}\right],
\end{array}
$$

or,

$$
\gamma_{0}=C_{0}+\sum_{v=1}^{p} C_{v} \gamma_{0} \Leftrightarrow\left(1-\sum_{v=1}^{p} C_{v}\right) \gamma_{0}=C_{0},
$$

where $C_{0}=C+\left(1+v_{1}\right) \widehat{C}>0$ and $C_{v}$ are the coefficients defined in the statement of the theorem. Hence, the previous equality implies $1-\sum_{v=1}^{p} C_{v}>0$, that is, the roots of the equation $1-C_{1} z-\ldots-C_{p} z^{p}=0$ lie outside the unit circle.

Let us point out that when $X$ follows a CP-INARCH $(p)$ model we easily obtain the constant $\widehat{C}=-2 \alpha_{0} \mu \sum_{v=1}^{p-1} \sum_{j-i=v} \alpha_{i} \alpha_{j} \sum_{u=1}^{p-1} d_{v u}>0$ (Appendix C). Therefore, in this case, we do not need to ensure that $\alpha_{0}\left(1+v_{1}\right)>v_{0}$ and the Theorem 3.4 assumes the following form.

Corollary 3.2 Let $X$ be a first order stationary process following a CP-INARCH $(p)$ model that satisfies $\boldsymbol{H}$ 2. If the process is second order stationary, then the equation $1-C_{1} z-\ldots-C_{p} z^{p}=0$ has all roots outside the unit circle, where for $u, l=1, \ldots, p-1$,

$$
\begin{gathered}
C_{u}=\left(1+v_{1}\right)\left[\alpha_{u}^{2}-\sum_{v=1}^{p-1} \sum_{|i-j|=v} \alpha_{i} \alpha_{j} d_{v u} b_{u 0}\right], \quad C_{p}=\left(1+v_{1}\right) \alpha_{p}^{2}, \\
b_{l 0}=\alpha_{l}, \quad b_{l l}=\sum_{|i-l|=l} \alpha_{i}-1 \text { and for } u \neq l, \quad b_{l u}=\sum_{|i-l|=u} \alpha_{i},
\end{gathered}
$$

with $B=\left(b_{i j}\right)$ and $B^{-1}=\left(d_{i j}\right)$ squared matrices of order $p-1$.

In the following we present some examples to illustrate the conditions of second order stationarity displayed.

Example 3.1 Let us consider a CP-INGARCH(2,2) model satisfying the hypothesis $\mathbf{H 2}$ and such that $\sum_{i=1}^{2}\left(\alpha_{i}+\beta_{i}\right)<1$. To examine the sufficient condition of second order stationarity we consider the polynomial matrix $P(z)=I_{3}-B_{1} z-B_{2} z^{2}$, with $B_{1}$ and $B_{2}$ the squared matrices of order 3 given by

$$
\begin{aligned}
B_{1} & =\left[\begin{array}{ccc}
\left(\alpha_{1}+\beta_{1}\right)^{2}+v_{1} \alpha_{1}^{2} & 2\left(1+v_{1}\right) \alpha_{2}\left(\alpha_{1}+\beta_{1}\right) & 2\left(1+v_{1}\right) \beta_{2}\left(\alpha_{1}+\beta_{1}\right) \\
\alpha_{1}+\frac{\beta_{1}}{1+v_{1}} & \alpha_{2} & \beta_{2} \\
\frac{\alpha_{1}+\beta_{1}}{1+v_{1}} & \alpha_{2} & \beta_{2}
\end{array}\right], \\
B_{2} & =\left[\begin{array}{ccc}
\left(\alpha_{2}+\beta_{2}\right)^{2}+v_{1} \alpha_{2}^{2} & 0 & 0 \\
0 & 0 & 0 \\
0 & 0 & 0
\end{array}\right] .
\end{aligned}
$$

Thus, the determinant of this polynomial is

$$
\begin{aligned}
\operatorname{det}(P(z))=1- & {\left[\left(\alpha_{1}+\beta_{1}\right)^{2}+\alpha_{2}+\beta_{2}+v_{1} \alpha_{1}^{2}\right] z } \\
& -\left[\left(\alpha_{1}+\beta_{1}\right)^{2}\left(\alpha_{2}+\beta_{2}\right)+\left(\alpha_{2}+\beta_{2}\right)^{2}+v_{1}\left(\alpha_{2}^{2}-\alpha_{1}^{2} \beta_{2}+\alpha_{1}^{2} \alpha_{2}+2 \alpha_{1} \alpha_{2} \beta_{1}\right)\right] z^{2} \\
& -\left[-\left(\alpha_{2}+\beta_{2}\right)^{3}-v_{1} \alpha_{2}^{2}\left(\alpha_{2}+\beta_{2}\right)\right] z^{3} .
\end{aligned}
$$


So

$$
\begin{gathered}
\operatorname{det}(P(1))>0 \Leftrightarrow\left(\alpha_{1}+\beta_{1}\right)^{2}\left(1+\alpha_{2}+\beta_{2}\right)+\left(\alpha_{2}+\beta_{2}\right)\left[1+\alpha_{2}+\beta_{2}-\left(\alpha_{2}+\beta_{2}\right)^{2}\right] \\
+v_{1}\left(\alpha_{1}^{2}\left[1+\alpha_{2}-\beta_{2}\right]+\alpha_{2}^{2}\left[1-\alpha_{2}-\beta_{2}\right]+2 \alpha_{1} \alpha_{2} \beta_{1}\right)<1,
\end{gathered}
$$

that is, whenever the coefficients of the model satisfy this inequality, the process $X$ is second order stationary. In order to obtain the necessary condition, we study the roots of the equation $1-C_{1} z-C_{2} z^{2}=0$ with

$$
\begin{aligned}
C_{1} & =\left(\alpha_{1}+\beta_{1}\right)^{2}+v_{1} \alpha_{1}^{2}-2\left(1+v_{1}\right)\left[\left(\alpha_{1}+\beta_{1}\right) \sum_{u=1}^{2}\left(\alpha_{2} d_{1 u}+\beta_{2} d_{2 u}\right) b_{u 0}\right] \\
& =\left(\alpha_{1}+\beta_{1}\right)^{2}+v_{1} \alpha_{1}^{2}+2\left(1+v_{1}\right)\left(\alpha_{1}+\beta_{1}\right) \frac{\alpha_{2} b_{10}+\beta_{2} b_{20}}{1-\alpha_{2}-\beta_{2}} \\
& =\left[\frac{1+\alpha_{2}+\beta_{2}}{1-\alpha_{2}-\beta_{2}}\right]\left(\alpha_{1}+\beta_{1}\right)^{2}+v_{1}\left[\frac{\alpha_{1}\left(1+\alpha_{2}-\beta_{2}\right)+2 \alpha_{2} \beta_{1}}{1-\alpha_{2}-\beta_{2}}\right] \alpha_{1}, \\
C_{2} & =\left(\alpha_{2}+\beta_{2}\right)^{2}+v_{1} \alpha_{2}^{2},
\end{aligned}
$$

since the matrices $B, B^{-1}$ and the vector $b$ are given by

$$
b=\left[\begin{array}{c}
\alpha_{1}+\frac{\beta_{1}}{1+v_{1}} \\
\frac{\alpha_{1}+\beta_{1}}{1+v_{1}}
\end{array}\right], \quad B=\left[\begin{array}{cc}
\alpha_{2}-1 & \beta_{2} \\
\alpha_{2} & \beta_{2}-1
\end{array}\right], \quad B^{-1}=\left[\begin{array}{cc}
\frac{\beta_{2}-1}{1-\alpha_{2}-\beta_{2}} & \frac{-\beta_{2}}{1-\alpha_{2}-\beta_{2}} \\
\frac{-\alpha_{2}}{1-\alpha_{2}-\beta_{2}} & \frac{\alpha_{2}-1}{1-\alpha_{2}-\beta_{2}}
\end{array}\right] .
$$

Hence, the roots of the equation are outside the unit circle if and only if $C_{1}+C_{2}<1$, which coincides with the sufficient condition (10) of weak stationarity. We can also deduce from the last result the necessary and sufficient condition for second order stationarity of a CP-INARCH(2) model. Indeed, if $\beta_{1}=\beta_{2}=0$ we obtain

$$
\left(1+v_{1}\right)\left[\alpha_{1}^{2}\left(1+\alpha_{2}\right)+\alpha_{2}^{2}\left(1-\alpha_{2}\right)\right]+\alpha_{2}<1 .
$$

Figures 2, 3 and 4 illustrate, for $v_{1}=0$, the first and second order stationarity regions of the CP-INARCH(2) model. The region plotted in Figure 4 is obviously the intersection of the first order stationarity region with the set of points $\left(\alpha_{1}, \alpha_{2}\right)$ satisfying the last condition.

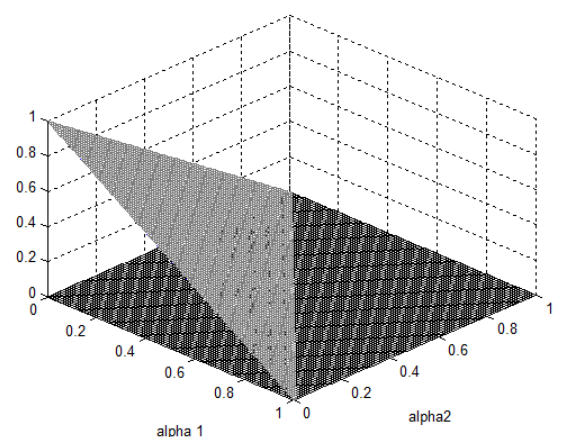

Figure 2: Frontier of the first order stationarity region of a $C P$ INARCH(2) model.

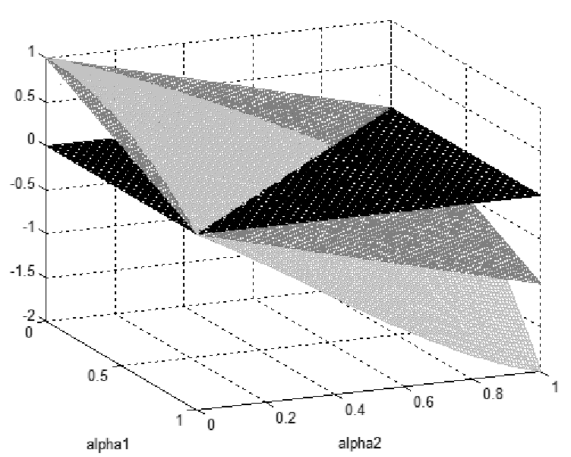

Figure 3: Frontiers of the first and second order stationarity regions of a $C P-I N A R C H(2)$ model. 


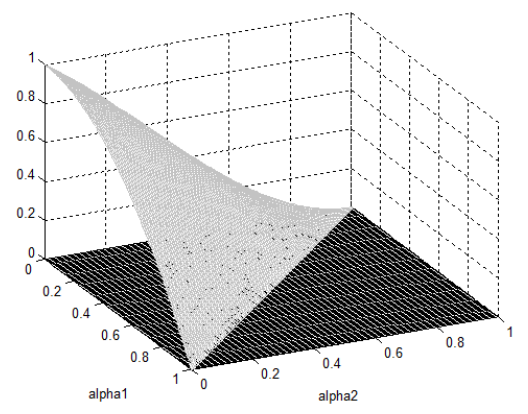

Figure 4: Frontier of the weak stationarity region of a $C P-I N A R C H(2)$ model.

The following example generalizes Theorem 3.3.

Example 3.2 Let us now consider a CP-INGARCH(p,p) model with $\alpha_{1}=\ldots=\alpha_{p-1}=\beta_{1}=$ $\ldots=\beta_{p-1}=0$ satisfying $\boldsymbol{H} 2$ and such that $\alpha_{p}+\beta_{p}<1$. To analyze the sufficient condition for weak stationarity of $X$ we consider the polynomial matrix resulting from the Theorem 3.2

$P(z)=I_{2 p-1}-B_{1} z-\ldots-B_{p} z^{p}$

$$
=\left[\begin{array}{cccccccccc}
1-\left[\left(\alpha_{p}+\beta_{p}\right)^{2}+v_{1} \alpha_{p}^{2}\right] z^{p} & 0 & 0 & \cdots & 0 & 0 & 0 & \cdots & 0 & 0 \\
0 & 1-\alpha_{p} z^{p-1} & -\alpha_{p} z^{p-2} & \cdots & -\alpha_{p} z & -\beta_{p} z^{p-1} & -\beta_{p} z^{p-2} & \cdots & -\beta_{p} z^{2} & -\beta_{p} z \\
0 & 0 & 1 & \cdots & 0 & 0 & 0 & \cdots & 0 & 0 \\
\vdots & \vdots & \vdots & \ddots & \vdots & \vdots & \vdots & \vdots & \vdots & \vdots \\
0 & 0 & 0 & \cdots & 1 & 0 & 0 & \cdots & 0 & 0 \\
0 & -\alpha_{p} z^{p-1} & -\alpha_{p} z^{p-2} & \cdots & -\alpha_{p} z & 1-\beta_{p} z^{p-1} & -\beta_{p} z^{p-2} & \cdots & -\beta_{p} z^{2} & -\beta_{p} z \\
0 & 0 & 0 & \cdots & 0 & 0 & 1 & \cdots & 0 & 0 \\
\vdots & \vdots & \vdots & \vdots & \vdots & \vdots & \vdots & \ddots & \vdots & \vdots \\
0 & 0 & 0 & \cdots & 0 & 0 & 0 & \cdots & 1 & 0 \\
0 & 0 & 0 & \cdots & 0 & 0 & 0 & \cdots & 0 & 1
\end{array}\right]
$$

with $B_{k}(k=1, \ldots, p)$ squared matrices of order $2 p-1$. In what follows denote by $P_{i j}(z)$ the submatrix of $P(z)$ obtained by deleting the row $i$ and the column $j$. Applying Laplace theorem to the first row of the matrix $P(z)$ we have

$$
\operatorname{det} P(z)=\left[1-\left(\left(\alpha_{p}+\beta_{p}\right)^{2}+v_{1} \alpha_{p}^{2}\right) z^{p}\right] \operatorname{det} P_{11}(z)
$$

with

$$
\begin{array}{r}
\operatorname{det} P_{11}(z)=\left(1-\alpha_{p} z^{p-1}\right) \operatorname{det} Q_{11}(z)+\alpha_{p} z^{p-2} \operatorname{det} Q_{12}(z)-\ldots+(-1)^{p+1} \alpha_{p} z \operatorname{det} Q_{1, p-1}(z) \\
+(-1)^{p+2} \beta_{p} z^{p-1} \operatorname{det} Q_{1 p}(z)+(-1)^{p+3} \beta_{p} z^{p-2} \operatorname{det} Q_{1, p+1}(z)+\ldots+\beta_{p} z \operatorname{det} Q_{1,2 p-2}(z),
\end{array}
$$

using again the Laplace theorem in the first row of the matrix $P_{11}(z)$ and taking $Q_{i j}(z)$ the submatrix of $P_{11}(z)$ obtained by deleting the row $i$ and the column $j$. Let us note that $\operatorname{det} Q_{12}(z)=$ $\ldots=\operatorname{det} Q_{1, p-1}(z)=\operatorname{det} Q_{1, p+1}(z)=\ldots=\operatorname{det} Q_{1,2 p-2}(z)=0$ because all the matrices have a null row. Now, applying Laplace theorem on row $p-1$ of the matrices $Q_{11}(z)$ and $Q_{1 p}(z)$ we obtain

$$
\begin{aligned}
& \operatorname{det} Q_{11}(z)=(-1)^{2 p-2}\left(1-\beta_{p} z^{p-1}\right), \\
& \operatorname{det} Q_{1 p}(z)=(-1)^{p+1} \alpha_{p} z^{p-1},
\end{aligned}
$$

since when we delete that line, a column of zeros appears except when we consider the term in the position $(p-1, p-1)$ and $(1,1)$, respectively. This allows us to conclude that

$$
\operatorname{det} P_{11}(z)=\left(1-\alpha_{p} z^{p-1}\right)\left(1-\beta_{p} z^{p-1}\right)+(-1)^{2 p+3} \alpha_{p} \beta_{p} z^{2 p-2}=1-\left(\alpha_{p}+\beta_{p}\right) z^{p-1}
$$


and finally

$$
\operatorname{det} P(z)=1-\left(\alpha_{p}+\beta_{p}\right) z^{p-1}-\left[\left(\alpha_{p}+\beta_{p}\right)^{2}+v_{1} \alpha_{p}^{2}\right]\left[1-\left(\alpha_{p}+\beta_{p}\right) z^{p-1}\right] z^{p} .
$$

We deduce that the sufficient condition for second order stationarity of the considered model, and taking into account that $\alpha_{p}+\beta_{p}<1$, is given by

$$
\operatorname{det}(P(1))>0 \Leftrightarrow\left(\alpha_{p}+\beta_{p}\right)^{2}+v_{1} \alpha_{p}^{2}<1 .
$$

Finally this condition is also the necessary condition obtained in Theorem 3.4 as it reduces to ensure that the roots of $1-C_{p} z^{p}=0$, with $C_{p}=\left(\alpha_{p}+\beta_{p}\right)^{2}+v_{1} \alpha_{p}^{2}$, lie outside the unit circle, that is, $C_{p}<1$. Let us note that in this case it is not necessary to ensure that $\alpha_{0}\left(1+v_{1}\right)>v_{0}$ because in the proof of Theorem 3.4 we have $\widehat{C}=0$ and so the constant $C_{0}$ is always positive.

\subsection{Strict stationarity}

In this section we study the existence of strictly stationary solutions for the class of models previously introduced. The study undertaken allows us to establish the existence of strictly stationary and ergodic processes in a subclass of CP-INGARCH $(p, q)$ models for which the characteristic functions $\varphi_{t}$ are deterministic. We stress that this particular case still includes a wide class of models not studied in literature. As an example we refer the GEOMP2-INGARCH model introduced in example 2.1.3. We begin by building a first order stationary process solution of the model that, under certain conditions, will be strictly stationary and ergodic.

\subsubsection{Construction of a process solution when $\varphi_{t}$ is deterministic}

Let us consider model (1) associated to a given family of characteristic functions $\left(\varphi_{t}, t \in \mathbb{Z}\right)$ such that the hypothesis $\boldsymbol{H} \mathbf{1}$ is satisfied. We assume $\boldsymbol{H} 3: \varphi_{t}$ is deterministic.

Let $\left(U_{t}, t \in \mathbb{Z}\right)$ be a sequence of independent real random variables distributed according to a discrete compound Poisson law with characteristic function

$$
\Phi_{U_{t}}(u)=\exp \left\{\frac{\alpha_{0}}{B(1)} \frac{i}{\varphi_{t}^{\prime}(0)}\left[\varphi_{t}(u)-1\right]\right\} .
$$

For each $t \in \mathbb{Z}$ and $k \in \mathbb{N}$, let $\mathcal{Z}_{t, k}=\left\{Z_{t, k, j}\right\}_{j \in \mathbb{N}}$ be a sequence of independent discrete compound Poisson random variables with characteristic function

$$
\Phi_{Z_{t, k, j}}(u)=\exp \left\{\psi_{k} \frac{i}{\varphi_{t+k}^{\prime}(0)}\left[\varphi_{t+k}(u)-1\right]\right\},
$$

where $\left(\psi_{j}, j \in \mathbb{N}\right)$ is the sequence of coefficients associated to the $\mathrm{CP}-\mathrm{INARCH}(\infty)$ representation of the model. We note that $E\left(U_{t}\right)=\alpha_{0} B^{-1}(1)=\psi_{0}, E\left(Z_{t, k, j}\right)=\psi_{k}$ and that $Z_{t, k, j}$ are identically distributed for each $(t, k) \in \mathbb{Z} \times \mathbb{N}$. We also assume that all the variables $U_{s}, Z_{t, k, j}$, $s, t \in \mathbb{Z}, k, j \in \mathbb{N}$, are mutually independent. Based on these random variables, we define the sequence $X_{t}^{(n)}$ as follows:

$$
X_{t}^{(n)}=\left\{\begin{array}{ll}
0, & n<0 \\
U_{t}, & n=0 \\
U_{t}+\sum_{k=1}^{n} \sum_{j=1}^{X_{t-k}^{(n-k)}} Z_{t-k, k, j}, & n>0
\end{array},\right.
$$

where it is assumed that $\sum_{j=1}^{0} Z_{t-k, k, j}=0$.

Let us recall the definition of thinning operation: considering a non-negative integer-valued random variable $W$ and $\phi \geq 0$, the thinning operation is defined by

$$
\phi \circ W=\left\{\begin{array}{ll}
\sum_{j=1}^{W} V_{j}, & \text { if } W>0 \\
0, & \text { otherwise }
\end{array},\right.
$$


where $\left\{V_{j}\right\}$, called counting series, is a sequence of i.i.d. non-negative integer-valued random variables, independent of $W$ and such that $E\left(V_{j}\right)=\phi$. An important property of this operation is that $E(\phi \circ W)=\phi E(W)$ (Gauthier and Latour, 1994).

Using this definition, $X_{t}^{(n)}, n>0$, is rewritten in the form

$$
X_{t}^{(n)}=U_{t}+\sum_{k=1}^{n} \psi_{k}^{(t-k)} \circ X_{t-k}^{(n-k)},
$$

where the notation $\left(\psi_{k}^{(\tau)} \circ\right)$ means that the sequence of random variables of mean $\psi_{k}$ involved in the thinning operation corresponds to time $\tau$.

In what follows we present some properties of the sequence $X_{t}^{(n)}$, which will be of interest in the study of its behavior.

Property 3.1 If $\sum_{i=1}^{p} \alpha_{i}+\sum_{j=1}^{q} \beta_{j}<1$ then $\left\{\left(X_{t}^{(n)}, t \in \mathbb{Z}\right), n \in \mathbb{Z}\right\}$ is a sequence of first order stationary processes such that, as $n \rightarrow \infty$,

$$
\mu_{n}=E\left(X_{t}^{(n)}\right) \longrightarrow \mu .
$$

Proof. We start by noting that $E\left(X_{t}^{(n)}\right)$ does not depend on $t, \forall n \in \mathbb{Z}$. The result is trivial for $n<0$. For $n=0$ we obtain $E\left(X_{t}^{(0)}\right)=E\left(U_{t}\right)=\psi_{0}$, which is also independent of $t$. Let us consider now, as induction hypothesis, that for an arbitrarily fixed value of $t$ and until $n>0$, $E\left(X_{t}^{(n)}\right)$ is independent of $t$. Therefore,

$$
E\left(X_{t}^{(n+1)}\right)=\psi_{0}+\sum_{k=1}^{n+1} \psi_{k} E\left(X_{t-k}^{(n+1-k)}\right)=g\left(E\left(X_{t-n-1}^{(0)}\right), \ldots, E\left(X_{t-1}^{(n)}\right)\right)
$$

that is, an independent function of $t$. So

$$
\mu_{n}=E\left(X_{t}^{(n)}\right)= \begin{cases}0, & n<0 \\ \psi_{0}, & n=0 \\ \psi_{0}+\sum_{k=1}^{n} \psi_{k} \mu_{n-k}, & n>0\end{cases}
$$

which for $n>0$ is equivalent to

$$
\mu_{n}=\sum_{k=1}^{\infty} \psi_{k} \mu_{n-k}+\psi_{0}=B^{-1}(L)\left[A(L) \mu_{n}+\alpha_{0}\right] \Leftrightarrow K(L) \mu_{n}=\alpha_{0},
$$

where $K(L)=B(L)-A(L)$. Thus, the sequence $\left\{\mu_{n}\right\}$ satisfies a finite difference equation of degree $\max (p, q)$ with constant coefficients. The characteristic polynomial $K(z)$ of this equation has all its roots outside the unit circle since $\sum_{i=1}^{p} \alpha_{i}+\sum_{j=1}^{q} \beta_{j}<1$, and so, $\left\{\left(X_{t}^{(n)}, t \in \mathbb{Z}\right), n \in \mathbb{Z}\right\}$ is a sequence of first order stationary processes. From this stationarity, we deduce

$$
\lim _{n \rightarrow \infty} \mu_{n}=\frac{\psi_{0}}{1-\sum_{k=1}^{\infty} \psi_{k}}=\frac{\alpha_{0} B^{-1}(1)}{1-H(1)}=\frac{\alpha_{0}}{K(1)}=\frac{\alpha_{0}}{1-\sum_{i=1}^{p} \alpha_{i}-\sum_{j=1}^{q} \beta_{j}}=\mu .
$$

Property 3.2 If $\sum_{i=1}^{p} \alpha_{i}+\sum_{j=1}^{q} \beta_{j}<1$ and $\varphi_{t}$ is derivable at zero up to order 2 , then the sequence $\left\{\left(X_{t}^{(n)}, t \in \mathbb{Z}\right), n \in \mathbb{Z}\right\}$ converges almost surely, in $L^{1}$ and $L^{2}$ to a process $X^{*}=\left(X_{t}^{*}, t \in \mathbb{Z}\right)$.

Proof. Let us begin by showing that $\left\{\left(X_{t}^{(n)}, t \in \mathbb{Z}\right), n \in \mathbb{Z}\right\}$ is a non-decreasing sequence. Indeed, when $n=0$ and for a fixed value of $t$, we have

$$
X_{t}^{(1)}-X_{t}^{(0)}=U_{t}+\sum_{j=1}^{X_{t-1}^{(0)}} Z_{t-1,1, j}-U_{t}=\sum_{j=1}^{U_{t-1}} Z_{t-1,1, j} \geq 0,
$$


because this is a random sum of non-negative integer random variables. Supposing that for any fixed $t$ and for $n>0$ we have $X_{t}^{(n)}-X_{t}^{(n-1)} \geq 0$ we obtain

$$
X_{t}^{(n+1)}-X_{t}^{(n)}=\sum_{k=1}^{n} \sum_{j=X_{t-k}^{(n-k)}+1}^{X_{t-k}^{(n+1-k)}} Z_{t-k, k, j}+\sum_{j=1}^{U_{t-n-1}} Z_{t-n-1, n+1, j},
$$

which is obviously a non-negative process. Using the monotony of the sequence and the hypothesis on the model coefficients we prove that $\left\{\left(X_{t}^{(n)}, t \in \mathbb{Z}\right), n \in \mathbb{Z}\right\}$ converges almost surely to a process, $\left(X_{t}^{*}, t \in \mathbb{Z}\right)$, that is almost surely finite, using Borel-Cantelli theorem like in Proposition 2 of Ferland et al. (2006). Applying Beppo Lévi's theorem we conclude that the first moment of $X_{t}^{*}$ is finite since according to Property 3.1

$$
\mu=\lim _{n \rightarrow \infty} \mu_{n}=\lim _{n \rightarrow \infty} E\left(X_{t}^{(n)}\right)=E\left(X_{t}^{*}\right),
$$

and consequently the convergence of $\left\{\left(X_{t}^{(n)}, t \in \mathbb{Z}\right), n \in \mathbb{Z}\right\}$ in $L^{1}$ is deduced. For its convergence in $L^{2}$, we proceed as Ferland et al. (2006) in Proposition 4 and 5, noting that

$$
V\left(Z_{t-k, k, j}\right)=-\Phi_{Z_{t-k, k, j}}^{\prime \prime}(0)-\psi_{k}^{2}=-i \frac{\varphi_{t}^{\prime \prime}(0)}{\varphi_{t}^{\prime}(0)} \psi_{k}<\infty
$$

\subsubsection{Stationarity and Ergodicity}

Taking into account the results of the previous section, we obtain the next lemma that will be useful to establish the existence of a strictly stationary and ergodic process satisfying (1).

Lemma 3.1 Under the hypothesis $\mathbf{H 3}$, the process $X^{*}$ is a solution of the $\operatorname{CP-INGARCH}(p, q)$ model if $\sum_{j=1}^{p} \alpha_{j}+\sum_{k=1}^{q} \beta_{k}<1$.

Proof. The almost sure limit of the sequence $\left(X_{t}^{(n)}\right)$ is a solution of the model since,

$$
\Phi_{X_{t}^{*} \mid \underline{X}_{t-1}^{*}}(u) \stackrel{(a)}{=} \lim _{n \rightarrow+\infty} \Phi_{n}(u) \stackrel{(b)}{=} e^{i \frac{\lambda_{t}}{\varphi^{\prime}(0)}[\varphi(u)-1]}, \quad u \in \mathbb{R}
$$

with $\Phi_{n}$ the characteristic function of the sequence $r_{t}^{(n)} \mid \underline{X}_{t-1}^{*}$, where

$$
r_{t}^{(n)}=U_{t}+\sum_{k=1}^{n} \sum_{j=1}^{X_{t-k}^{*}} Z_{t-k, k, j}
$$

In fact, the equality (a) follows from Paul Lévy theorem since, similarly to section 2.6 of Ferland et al. (2006), we prove that for a fixed $t$, the sequence $Y_{t}^{(n)}=r_{t}^{(n)}-X_{t}^{(n)}$ converges in mean to zero, when $n \rightarrow \infty$. So $Y_{t}^{(n)}$ and $X_{t}^{*}-X_{t}^{(n)}$ converge in probability to zero which, using

$$
X_{t}^{*}-r_{t}^{(n)}=\left(X_{t}^{*}-X_{t}^{(n)}\right)+\left(X_{t}^{(n)}-r_{t}^{(n)}\right)=\left(X_{t}^{*}-X_{t}^{(n)}\right)-Y_{t}^{(n)},
$$

allows us to conclude that the sequence $r_{t}^{(n)}$ converges in probability to $X_{t}^{*}$ and then $r_{t}^{(n)} \mid \underline{X}_{t-1}^{*}$ converges in law to $X_{t}^{*} \mid \underline{X}_{t-1}^{*}$.

Let us obtain $\Phi_{n}$. Conditionally to $\underline{X}_{t-1}^{*}$, we have

$$
\begin{aligned}
\Phi_{\sum_{j=1}^{X_{t-k}^{*}} Z_{t-k, k, j}}(u) & =\prod_{j=1}^{X_{t-k}^{*}} \Phi_{Z_{t-k, k, j}}(u)=\exp \left\{\sum_{j=1}^{X_{t-k}^{*}} \psi_{k} \frac{i}{\varphi_{t}^{\prime}(0)}\left[\varphi_{t}(u)-1\right]\right\} \\
& =\exp \left\{\psi_{k} X_{t-k}^{*} \frac{i}{\varphi_{t}^{\prime}(0)}\left[\varphi_{t}(u)-1\right]\right\} .
\end{aligned}
$$


From the independence of the variables involved in the definition of $r_{t}^{(n)}$, we obtain

$$
\begin{aligned}
\Phi_{n}(u) & =\exp \left(\frac{\alpha_{0}}{B(1)} \frac{i}{\varphi_{t}^{\prime}(0)}\left[\varphi_{t}(u)-1\right]+\sum_{k=1}^{n} \psi_{k} X_{t-k}^{*} \frac{i}{\varphi_{t}^{\prime}(0)}\left[\varphi_{t}(u)-1\right]\right) \\
& =\exp \left\{\left(\frac{\alpha_{0}}{B(1)}+\sum_{k=1}^{n} \psi_{k} X_{t-k}^{*}\right) \frac{i}{\varphi_{t}^{\prime}(0)}\left[\varphi_{t}(u)-1\right]\right\},
\end{aligned}
$$

and thus, when $n \rightarrow \infty$, we have the equality $(b)$.

Observation 3.2 As a consequence of Theorem 3.1 and the previous lemma, the process $X^{*}$ is, under the hypothesis $\mathbf{H 3}$, a first order stationary solution of the CP-INGARCH $(p, q)$ model if $\sum_{j=1}^{p} \alpha_{j}+\sum_{k=1}^{q} \beta_{k}<1$.

Now, let us consider, additionally to the hypothesis $\mathbf{H 3}$, that $\varphi_{t}$ is independent of $t$. In this subclass, it is possible to establish the strict stationarity of $\left(X_{t}^{*}\right)$, generalizing the corresponding study of Ferland et al. (2006), as well as its ergodicity.

Theorem 3.5 Let us consider the model CP-INGARCH(p,q) defined by (1) with $\varphi_{t}, t \in \mathbb{Z}$, deterministic and independent of $t$.

(a) $\left\{\left(X_{t}^{(n)}, t \in \mathbb{Z}\right), n \in \mathbb{Z}\right\}$ is a sequence of strictly stationary and ergodic processes.

(b) There is a strictly stationary and ergodic process in $L^{1}$ that satisfies the model (1), if and only if $\sum_{i=1}^{p} \alpha_{i}+\sum_{j=1}^{q} \beta_{j}<1$. Moreover, its first two moments are finite.

Proof. (a) The proof of strict stationarity follows the procedure presented in Proposition 3 of Ferland et al. (2006), since in this case the sequences $\left(U_{t}, t \in \mathbb{Z}\right)$ and $\left(\mathcal{Z}_{t, k}, t \in \mathbb{Z}, k \in \mathbb{N}\right)$, defined in section 3.3.1, are of i.i.d. random variables. Moreover, $\left(X_{t}^{(n)}\right)$ is a sequence of ergodic processes, because it is a measurable function of the sequence of i.i.d. random variables $\left\{\left(U_{t}, \mathcal{Z}_{t, j}\right), t \in \mathbb{Z}, j \in\right.$ $\mathbb{N}$ \} (Durrett, 2010). In fact, from (12) and using recursively the thinning operator, $\left(X_{t}^{(n)}\right)$ is deduced from $\left(U_{t}, \mathcal{Z}_{t, j}\right)$ as we illustrate in the following for $n=1,2$ :

$$
\begin{aligned}
X_{t}^{(1)} & =U_{t}+\psi_{1}^{(t-1)} \circ X_{t-1}^{(0)}=U_{t}+\psi_{1}^{(t-1)} \circ U_{t-1}, \\
X_{t}^{(2)} & =U_{t}+\psi_{1}^{(t-1)} \circ X_{t-1}^{(1)}+\psi_{2}^{(t-2)} \circ X_{t-2}^{(0)} \\
& =U_{t}+\psi_{1}^{(t-1)} \circ\left(U_{t-1}+\psi_{1}^{(t-2)} \circ U_{t-2}\right)+\psi_{2}^{(t-2)} \circ U_{t-2},
\end{aligned}
$$

and so on, like in Ferland et al. (2006).

(b) In Lemma 3.1 we proved that $\left(X_{t}^{*}, t \in \mathbb{Z}\right)$ is a solution of (1). So, it is enough to prove that when $\varphi_{t}$ is deterministic and independent of $t$, the almost sure limit is strictly stationary and ergodic. From (a), $\left(X_{t}^{(n)}\right)$ is a sequence of strictly stationary processes. Otherwise, $\left(X_{t}^{(n)}\right)$ converges almost surely to $\left(X_{t}^{*}\right)$ if $\sum_{i=1}^{p} \alpha_{i}+\sum_{j=1}^{q} \beta_{j}<1$. So, considering without loss of generality, the indexes $\{1, \ldots, k\}$, we have

$$
\left(X_{1}^{(n)}, \ldots, X_{k}^{(n)}\right) \longrightarrow_{n \rightarrow+\infty}\left(X_{1}^{*}, \ldots, X_{k}^{*}\right), \quad\left(X_{1+h}^{(n)}, \ldots, X_{k+h}^{(n)}\right) \longrightarrow_{n \rightarrow+\infty}\left(X_{1+h}^{*}, \ldots, X_{k+h}^{*}\right),
$$

almost surely, for any $h \in \mathbb{Z}$, and consequently, in law. Considering the strict stationarity of $\left(X_{t}^{(n)}\right)$ and the limit unicity, it is easy to conclude that $\left(X_{t}^{*}\right)$ is a strictly stationary process. Moreover, taking into account that $\left(X_{t}^{(n)}\right)$ is the measurable function of $\left(U_{t}, \mathcal{Z}_{t, j}\right)$ referred above, i.e.,

$$
X_{t}^{(n)}=T_{n}\left(U_{t}, \ldots, U_{t-n}, Z_{t-1,1, k}, \ldots, Z_{t-n, n, k}, k \in \mathbb{N}\right),
$$

with $T_{n}$ a sequence of measurable functions, $\left(X_{t}^{*}\right)$ may be written as the almost sure limit of (13), that is,

$$
X_{t}^{*}=T\left(\left(U_{t}, Z_{t, j, k}\right), t \in \mathbb{Z}, j, k \in \mathbb{N}\right),
$$


where $T$ is the almost sure limit of $T_{n}$, and so a measurable function (Halmos, 1974). Thus $\left(X_{t}^{*}\right)$ is ergodic since it is also a measurable function of the ergodic process $\left(\left(U_{t}, Z_{t, j, k}\right), t \in \mathbb{Z}, j, k \in \mathbb{N}\right)$ (Billingsley, 1995, Theorem 36.4).

Regarding the necessary condition, we observe that if $\left(X_{t}\right)$ is a strictly stationary solution of model (1) it is also first order stationary as, by hypothesis, it is a process of $L^{1}$. So, by Theorem 3.1 we have $\sum_{i=1}^{p} \alpha_{i}+\sum_{j=1}^{q} \beta_{j}<1$.

Observation 3.3 Under the conditions of the previous theorem it follows that $\left\{X_{t}^{*}\right\}_{t \in \mathbb{Z}}$ is also a weakly stationary solution of the model because it is a strictly stationary second order process.

\section{Conclusion}

In this paper a general class of INGARCH models was introduced, including as particular cases some recent contributions on the modeling of integer-valued time series (Ferland et al., 2006, Zhu, 2011, 2012a). This generality is achieved considering that the distribution of $X_{t}$ given its past belongs to the family of infinitely divisible discrete laws and defining the model by means of the corresponding characteristic function. We are in presence of a wide class of discrete distributions which includes several well-known examples in literature such as the logarithmic and Borel laws (Johnson et al., 2005). Nevertheless, to use the model presented for some compound Poisson distributions may be nontrivial due to the difficulty in obtaining the equivalent representation considered in our definition. For instance, the zero-inflated geometric INGARCH model, already studied by Zhu (2012b), can be included in our framework (Aghababaei Jazi and Alamatsaz, 2011) but, to the best of the authors' knowledge, more studies must be done to exhibit that representation. A new model derived directly from this general framework is the GEOMP-INGARCH one. We note that this model is naturally interesting in practice as the associated conditional distribution, the geometric Poisson, is particularly useful in the study of the traffic accident data (see Özel and İnal, 2010, and the references therein for more examples). In what concerns this matter, we point out that more than to present a model for which the conditional distribution is compound Poisson, we can define it clearly as a counting process by introducing its generator process.

Conditions for first and second order stationarity are given and the existence of a strictly stationary and ergodic solution is established in a large subclass which includes, in particular, the Poisson and generalized Poisson INGARCH models. Moreover, we are strongly convinced that the sufficient condition of second order stationarity is also a necessary one as in the cases developed in examples 3.1 and 3.2 .

The probabilistic study developed here will be very useful in future statistical studies as, in particular, those related to the model estimation. Other probabilistic studies may be considered in future as, for instance, those of moments greater than 2 which will be essential in the evaluation of other features of the model like leptokurtosis or Taylor property (Gonçalves, Leite and Mendes-Lopes, 2009). Moreover, the analysis of the strict stationarity of this model when $\varphi_{t}$ is a random function is still an open question that deserves further development. According to the study presented in Fokianos and Christou (2012) on the mixed Poisson processes, to explore relationships between those models and CP-INGARCH ones seems to be useful for the statistical developments of this new class. Despite all this future work in order to implement these general models in practice, we should stress that the studies developed here, unifying and enlarging several approaches recently considered in the literature, present a significant contribution to the modeling of integer-valued time series.

\section{Acknowledgements}

The authors express their gratitude to the anonymous referees and the Co-Editor for their valuable suggestions and comments which have significantly improved the paper. 
This work is supported by the Centro de Matemática da Universidade de Coimbra (funded by the European Regional Development Fund through the program COMPETE and by the Portuguese Government through the FCT - Fundação para a Ciência e a Tecnologia under the projects PEst-C/MAT/UI0324/2011). The work of the third author was supported by a grant from the FCT with reference SFRH/BD/85336/2012.

\section{References}

[1] Aghababaei Jazi, M., Alamatsaz, M. H., Some contributions to inflated generalized power series distributions, Pak. J. Statist., Vol. 27(2), 139-157, 2011.

[2] Billingsley, P., Probability and Measure, New York, Wiley, 3rd ed., 1995.

[3] Bollerslev, T., Generalized autoregressive conditional heteroscedasticity, Journal of Econometrics, $37,307-327,1986$.

[4] Consul, P. C., Famoye, F., Lagrangian Probability Distributions, Birkhäuser, Boston, 2006.

[5] Doukhan, P., Latour, A. , Oraichi, D., A simple Integer-Valued bilinear time series model, Adv. Appl. Prob., 38, 559-578, 2006.

[6] Drost, F., van den Akker, R., Werker, B. J. M., Note on Integer-Valued bilinear time series models, Stat. and Prob. Letters, 78, 992-996, 2008.

[7] Durrett, R., Probability: Theory and Examples, Cambridge University Press, 4th ed., 2010.

[8] Ferland, R., Latour, A. and Oraichi, D., Integer-valued GARCH process, Journal of Time Series Analysis 27, 923-42, 2006.

[9] Fokianos, K., Christou, V., Quasi-likelihood inference for mixed Poisson time series, COMPSTAT 2012 Proceedings, 2012.

[10] Fokianos, K., Rahbek, A. and Tjøtheim, D., Poisson autoregression, Journal of the American Statistical Association 104, 1430-39, 2009.

[11] Gauthier, G. and Latour, A., Convergence forte des estimateurs des paramètres d'un processus GENAR(p), Annales des Sciences Mathématiques du Québec, 18, 49-71, 1994.

[12] Goldberg, S., Introduction to Difference Equations, New York: Wiley, 1958.

[13] Gonçalves, E., Leite, J. and Mendes-Lopes, N., A mathematical approach to detect the Taylor property in TARCH processes, Stat. and Prob. Letters 72, 602-610, 2009.

[14] Gut, A., Some remarks on the Riemann zeta distribution, Rev. Roumaine Math. Pures Appl. 51, 205-217, 2006.

[15] Halmos, P., Measure Theory, Springer-Verlag, New York, 1974.

[16] Horn, R. A., and Jonhson, C. R., Matrix Analysis, Cambridge University Press, New York, 2nd ed., 2013.

[17] Johnson, N.L., Kotz, S. and Kemp, A.W., Univariate discrete distributions, Wiley, New York, 3rd ed., 2005.

[18] Özel, G., and İnal, C., The probability function of a geometric Poisson distribution, Journal of Statistical Computation and Simulation 80 5, 479-487, 2010.

[19] Quarteroni, A., Sacco, R., and Saleri, F., Numerical Mathematics, Texts in Applied Mathematics 37, Springer-Verlag, New York, 2000.

[20] Steutel, F. W., and van Harn, K., Infinite divisibility of probability distributions on the real line, Marcel-Dekker, Inc, New York, 2004. 
[21] Weiß, C. H., Thinning operations for modelling time series of counts- a survey, Adv. Stat. Anal. 92, 319-341, 2008.

[22] Weiß, C. H., Modelling time series of counts with overdispersion, Statistical Methods and Applications 18, 507-519, 2009.

[23] Xu, H-Y., Xie, M., Goh, T. N. and Fu, X., A model for integer-valued time series with conditional overdispersion, Computational Statistics and Data Analysis, 56, 4229-4242, 2012.

[24] Zhu, F., A negative binomial integer-valued GARCH model, J. Time Ser. Anal. 32, 54-67, 2011.

[25] Zhu, F., Modelling overdispersed or underdispersed count data with generalized Poisson integer-valued GARCH models, Journal of Mathematical Analysis and Applications, 389 1, 58-71, 2012a.

[26] Zhu, F., Zero-inflated Poisson and negative binomial integer-valued GARCH models, Journal of Stat. Plann. and Inf., 142, 826-839, 2012b.

[27] Zhu, F., Li, Q. and Wang, D., A mixture integer-valued ARCH model, Journal of Stat. Plann. and Inf., 140, 2025-2036, 2010.

\section{A Some notation and assumptions}

We summarize the most important notation and the assumptions that are used in this paper:

$\Phi_{X}$ denotes the characteristic function of $X$ and $\Phi_{X}^{\prime \prime}$ is its second order derivative.

$\varphi_{t}$ is the characteristic function of the compounding distribution in the compound Poisson law and $\varphi_{t}^{\prime}$ and $\varphi_{t}^{\prime \prime}$ are, respectively, its first and second-order derivative.

i.i.d. is the abbreviation of independent and identically distributed.

$I_{n}$ is the identity matrix of order $n$ and $e_{j}$ its order $j$ row.

$\lambda_{t}^{*}=i \frac{\lambda_{t}}{\varphi_{t}^{\prime}(0)}$ is the parameter of the Poisson law of the random variable $N_{t}$.

H1: $\sum_{j=1}^{q} \beta_{j}<1$.

H2: $-i \frac{\varphi_{t}^{\prime \prime}(0)}{\varphi_{t}^{\prime}(0)}=v_{0}+v_{1} \lambda_{t}$, with $v_{0} \geq 0, v_{1} \geq 0$, not simultaneously zero.

H3: $\varphi_{t}$ is deterministic.

\section{B Autoregressive equation of $W_{t}$}

From (4), (5) and (6) it follows that the vector $W_{t}$ satisfies the autoregressive equation of order $p, W_{t}=B_{0}+\sum_{k=1}^{p} B_{k} W_{t-k}$ where $B_{0}=\left(b_{j}\right)$ is such that

$$
b_{j}= \begin{cases}C, & j=1 \\ \mu\left(\alpha_{0}-\frac{v_{0} \beta_{j-1}}{v}\right), & j=2, \ldots, p \\ \mu\left(\alpha_{0}-\frac{v_{0}\left(\alpha_{j-p}+\beta_{j-p}\right)}{v}\right), & j=p+1, \ldots, 2 p-1\end{cases}
$$

with $v=1+v_{1}$, and $B_{k}(k=1, \ldots, p)$ are the squared matrices having generic element $b_{i j}^{(k)}$ given by:

- $\operatorname{row} i=1$ :

$$
b_{1 j}^{(k)}= \begin{cases}v \alpha_{k}^{2}+2 \alpha_{k} \beta_{k}+\beta_{k}^{2}, & j=1 \\ 2 v\left(\alpha_{k}+\beta_{k}\right) \alpha_{j+k-1}, & j=2, \ldots, p \\ 2 v\left(\alpha_{k}+\beta_{k}\right) \beta_{j+k-p}, & j=p+1, \ldots, 2 p-1\end{cases}
$$


- $\operatorname{row} i=k+1,(k \neq p)$ :

$$
b_{k+1, j}^{(k)}= \begin{cases}\alpha_{k}+\frac{\beta_{k}}{v}, & j=1 \\ \alpha_{j+k-1}, & j=2, \ldots, p \\ \beta_{j+k-p}, & j=p+1, \ldots, 2 p-1\end{cases}
$$

- $\operatorname{row} i=k+p$ :

$$
b_{k+p, j}^{(k)}= \begin{cases}\frac{\alpha_{k}+\beta_{k}}{v}, & j=1 \\ \alpha_{j+k-1}, & j=2, \ldots, p \\ \beta_{j+k-p}, & j=p+1, \ldots, 2 p-1\end{cases}
$$

- $\operatorname{row} i=k+j$ :

$$
b_{k+j, j}^{(k)}= \begin{cases}\alpha_{k}+\beta_{k}, & j=2, \ldots, p-k, p+1, \ldots, 2 p-1-k \\ 0 & j=p-k+1, \ldots, p\end{cases}
$$

and for any other case $b_{i j}^{(k)}=0$, where we consider $\alpha_{i}=\beta_{i}=0$, for $i>p$. The general form of these matrices is

$$
B_{k}=\left[\begin{array}{ll}
B_{1,1}^{(k)} & B_{1,2}^{(k)} \\
B_{2,1}^{(k)} & B_{2,2}^{(k)}
\end{array}\right],
$$

where $B_{1,1}^{(k)}$ is the squared matrix of order $p$,

$$
\begin{aligned}
& B_{1,1}^{(1)}=\left[\begin{array}{ccccc}
v \alpha_{1}^{2}+2 \alpha_{1} \beta_{1}+\beta_{1}^{2} & 2 v \alpha_{2}\left(\alpha_{1}+\beta_{1}\right) & \cdots & 2 v \alpha_{p-1}\left(\alpha_{1}+\beta_{1}\right) & 2 v \alpha_{p}\left(\alpha_{1}+\beta_{1}\right) \\
\alpha_{1}+\frac{\beta_{1}}{v} & \alpha_{2} & \cdots & \alpha_{p-1} & \alpha_{p} \\
0 & \alpha_{1}+\beta_{1} & \cdots & 0 & 0 \\
\vdots & \vdots & \ddots & \vdots & \vdots \\
0 & 0 & \cdots & \alpha_{1}+\beta_{1} & 0
\end{array}\right], \\
& B_{1,1}^{(2)}=\left[\begin{array}{cccccc}
v \alpha_{2}^{2}+2 \alpha_{2} \beta_{2}+\beta_{2}^{2} & 2 v \alpha_{3}\left(\alpha_{2}+\beta_{2}\right) & \ldots & 2 v \alpha_{p-1}\left(\alpha_{2}+\beta_{2}\right) & 2 v \alpha_{p}\left(\alpha_{2}+\beta_{2}\right) & 0 \\
0 & 0 & \ldots & 0 & 0 & 0 \\
\alpha_{2}+\frac{\beta_{2}}{v} & \alpha_{3} & \ldots & \alpha_{p-1} & \alpha_{p} & 0 \\
0 & \alpha_{2}+\beta_{2} & \cdots & 0 & 0 & 0 \\
\vdots & \vdots & \ddots & \vdots & \vdots & \vdots \\
0 & 0 & \cdots & \alpha_{2}+\beta_{2} & 0 & 0
\end{array}\right] \\
& B_{1,1}^{(p)}=\left[\begin{array}{cccc}
v \alpha_{p}^{2}+2 \alpha_{p} \beta_{p}+\beta_{p}^{2} & 0 & \cdots & 0 \\
0 & 0 & \cdots & 0 \\
\vdots & \vdots & \ddots & \vdots \\
0 & 0 & \cdots & 0
\end{array}\right],
\end{aligned}
$$

$B_{1,2}^{(k)}$ is the $p \times(p-1)$ matrix,

$$
\begin{aligned}
B_{1,2}^{(1)} & =\left[\begin{array}{cccc}
2 v \beta_{2}\left(\alpha_{1}+\beta_{1}\right) & \cdots & 2 v \beta_{p-1}\left(\alpha_{1}+\beta_{1}\right) & 2 v \beta_{p}\left(\alpha_{1}+\beta_{1}\right) \\
\beta_{2} & \cdots & \beta_{p-1} & \beta_{p} \\
0 & \cdots & 0 & 0 \\
\vdots & \ddots & \vdots & \vdots \\
0 & \cdots & 0 & 0
\end{array}\right], \\
B_{1,2}^{(2)} & =\left[\begin{array}{ccccc}
2 v \beta_{3}\left(\alpha_{2}+\beta_{2}\right) & \cdots & 2 v \beta_{p-1}\left(\alpha_{2}+\beta_{2}\right) & 2 v \beta_{p}\left(\alpha_{2}+\beta_{2}\right) & 0 \\
0 & \cdots & 0 & 0 & 0 \\
\beta_{3} & \cdots & \beta_{p-1} & \beta_{p} & 0 \\
0 & \cdots & 0 & 0 & 0 \\
\vdots & \ddots & \vdots & \vdots & \vdots \\
0 & \cdots & 0 & 0 & 0
\end{array}\right], \cdots, B_{1,2}^{(p)}=\mathbf{0},
\end{aligned}
$$


$B_{2,1}^{(k)}$ is the $(p-1) \times p$ matrix,

$$
B_{2,1}^{(1)}=\left[\begin{array}{ccccc}
\frac{\alpha_{1}+\beta_{1}}{v} & \alpha_{2} & \cdots & \alpha_{p-1} & \alpha_{p} \\
0 & 0 & \cdots & 0 & 0 \\
\vdots & \vdots & \ddots & \vdots & \vdots \\
0 & 0 & \cdots & 0 & 0
\end{array}\right], B_{2,1}^{(2)}=\left[\begin{array}{cccccc}
0 & 0 & \cdots & 0 & 0 & 0 \\
\frac{\alpha_{2}+\beta_{2}}{v} & \alpha_{3} & \cdots & \alpha_{p-1} & \alpha_{p} & 0 \\
0 & 0 & \cdots & 0 & 0 & 0 \\
\vdots & \vdots & \ddots & \vdots & \vdots & \vdots \\
0 & 0 & \cdots & 0 & 0 & 0
\end{array}\right], \cdots, B_{2,1}^{(p)}=\mathbf{0},
$$

and $B_{2,2}^{(k)}$ is the squared matrix of order $p-1$,

$$
B_{2,2}^{(1)}=\left[\begin{array}{cccc}
\beta_{2} & \cdots & \beta_{p-1} & \beta_{p} \\
\alpha_{1}+\beta_{1} & \cdots & 0 & 0 \\
\vdots & \ddots & \vdots & \vdots \\
0 & \cdots & \alpha_{1}+\beta_{1} & 0
\end{array}\right], B_{2,2}^{(2)}=\left[\begin{array}{ccccc}
0 & \cdots & 0 & 0 & 0 \\
\beta_{3} & \cdots & \beta_{p-1} & \beta_{p} & 0 \\
\alpha_{2}+\beta_{2} & \cdots & 0 & 0 & 0 \\
\vdots & \ddots & \vdots & \vdots & \vdots \\
0 & \cdots & \alpha_{2}+\beta_{2} & 0 & 0
\end{array}\right], \cdots, B_{2,2}^{(p)}=\mathbf{0} .
$$

\section{Invertibility of the matrix $B$ and positivity of $\widehat{C}$}

By definition, a matrix $B=\left(b_{i j}\right) \in \mathbb{R}^{(2 p-2) \times(2 p-2)}$ is strictly diagonally dominant by rows when

$$
\left|b_{i i}\right|>\sum_{\substack{j=1 \\ j \neq i}}^{2 p-2}\left|b_{i j}\right|, i=1, \ldots, 2 p-2 .
$$

As the process $X$, being second order stationary, is also first order stationary, we have from Theorem 3.1

$$
\begin{gathered}
\sum_{l=1}^{p}\left(\alpha_{l}+\beta_{l}\right)<1 \Leftrightarrow\left(\alpha_{2 i}+\beta_{2 i}\right)+\sum_{|l-i| \neq i}\left(\alpha_{l}+\beta_{l}\right)<1 \\
\Rightarrow\left\{\begin{array}{l}
\left|\alpha_{2 i}-1\right|>\beta_{2 i}+\sum_{|l-i| \neq i}\left(\alpha_{l}+\beta_{l}\right)-\left(\alpha_{i}+\beta_{i}\right), \quad \text { if } i=1, \ldots, p-1 \\
\left|\beta_{2 i}-1\right|>\alpha_{2 i}+\sum_{|l-i| \neq i}\left(\alpha_{l}+\beta_{l}\right)-\left(\alpha_{i}+\beta_{i}\right), \quad \text { if } i=p, \ldots, 2 p-2
\end{array},\right.
\end{gathered}
$$

that is, $B$ is strictly diagonally dominant by rows. From Levy-Desplanques theorem (Horn and Jonhson, 2013, pp. 352, 392) we know that a strictly diagonally dominant by rows matrix admits inverse. In addition, as $B$ is strictly diagonally dominant by rows, the same happens to $-B$. As $-b_{l u} \leq 0$, for $u \neq l$, and $-b_{l l}>0$ we conclude that $-B$ is a M-matrix (Quarteroni et al., 2000, p. 30), that is, $(-B)^{-1} \geq 0 \Rightarrow B^{-1} \leq 0 \Rightarrow d_{i j} \leq 0$. This allows us to conclude that the constant $\widehat{C}$ given by

$$
\begin{aligned}
\widehat{C}= & -2 \alpha_{0} \mu \sum_{v=1}^{p-1} \sum_{j=i=v}\left(\alpha_{i}+\beta_{i}\right)\left[\alpha_{j} \sum_{u=1}^{2 p-2} d_{v u}+\beta_{j} \sum_{u=1}^{2 p-2} d_{v+p-1, u}\right] \\
& +2 \frac{v_{0} \mu}{1+v_{1}} \sum_{v=1}^{p-1} \sum_{j-i=v}\left(\alpha_{i}+\beta_{i}\right)\left[\alpha_{j} \sum_{u=1}^{p-1} \beta_{u} d_{v u}+\alpha_{j} \sum_{u=p}^{2 p-2}\left(\alpha_{u-p+1}+\beta_{u-p+1}\right) d_{v u}\right. \\
& \left.+\beta_{j} \sum_{u=1}^{p-1} \beta_{u} d_{v+p-1, u}+\beta_{j} \sum_{u=p}^{2 p-2}\left(\alpha_{u-p+1}+\beta_{u-p+1}\right) d_{v+p-1, u}\right]
\end{aligned}
$$

is positive, under the assumption that $\alpha_{0}\left(1+v_{1}\right)>v_{0}$. 